\title{
Glutaredoxin 5 deficiency causes sideroblastic anemia by specifically impairing heme biosynthesis and depleting cytosolic iron in human erythroblasts
}

\author{
Hong Ye, ${ }^{1}$ Suh Young Jeong, ${ }^{1}$ Manik C. Ghosh, ${ }^{1}$ Gennadiy Kovtunovych, ${ }^{1}$ Laura Silvestri, ${ }^{2}$ \\ Danilo Ortillo, ${ }^{3}$ Naoya Uchida, ${ }^{4}$ John Tisdale, ${ }^{4}$ Clara Camaschella, ${ }^{2}$ and Tracey A. Rouault ${ }^{1}$ \\ ${ }^{1}$ Molecular Medicine Program, National Institute of Child Health and Human Development, NIH, Bethesda, Maryland, USA. \\ 2Università Vita-Salute e Istituto di Ricerca e Cura a Carattere Scientifico (IRCCS) San Raffaele, Milan, Italy. ${ }^{3}$ Department of Physics, \\ Emory University, Atlanta, Georgia, USA. ${ }^{4}$ Molecular and Clinical Hematology Branch, NHLBI, NIH, Bethesda, Maryland, USA.
}

\begin{abstract}
Glutaredoxin 5 (GLRX5) deficiency has previously been identified as a cause of anemia in a zebrafish model and of sideroblastic anemia in a human patient. Here we report that GLRX5 is essential for iron-sulfur cluster biosynthesis and the maintenance of normal mitochondrial and cytosolic iron homeostasis in human cells. GLRX5, a mitochondrial protein that is highly expressed in erythroid cells, can homodimerize and assemble [2Fe-2S] in vitro. In GLRX5-deficient cells, [Fe-S] cluster biosynthesis was impaired, the iron-responsive element-binding (IRE-binding) activity of iron regulatory protein 1 (IRP1) was activated, and increased IRP2 levels, indicative of relative cytosolic iron depletion, were observed together with mitochondrial iron overload. Rescue of patient fibroblasts with the WT GLRX5 gene by transfection or viral transduction reversed a slow growth phenotype, reversed the mitochondrial iron overload, and increased aconitase activity. Decreased aminolevulinate $\delta$, synthase 2 (ALAS2) levels attributable to IRP-mediated translational repression were observed in erythroid cells in which GLRX5 expression had been downregulated using siRNA along with marked reduction in ferrochelatase levels and increased ferroportin expression. Erythroblasts express both IRP-repressible ALAS2 and non-IRP-repressible ferroportin 1b. The unique combination of IRP targets likely accounts for the tissue-specific phenotype of human GLRX5 deficiency.
\end{abstract}

\section{Introduction}

Iron-sulfur clusters [Fe-S] are prosthetic groups that facilitate a wide range of cellular activities, including electron transfer, enzymatic catalysis, and sensing of iron and oxygen $(1,2)$. More than 20 proteins are involved in biogenesis of [Fe-S] clusters (3-7). Many $[\mathrm{Fe}-\mathrm{S}]$ biogenesis proteins are located primarily in mitochondria, including the sulfur donor ISCS proteins, the proposed iron donor frataxin, multiple scaffolds including ISCU and NFU as well as molecular chaperones, and redox proteins. [Fe-S] clusters in cytosol are also synthesized by a cytosolic machinery $(4,6,8,9)$, which in mammalian cells includes alternative isoforms of the sulfur donor, ISCS (10), the major scaffold protein, $\operatorname{ISCU}(8,9)$, and a possible alternative scaffold, NFU (11). In humans, because of the presence of iron regulatory protein 1 (IRP1), regulation of cellular iron homeostasis is linked to the [Fe-S] biosynthesis machinery (12-16). When IRP1 contains an [Fe-S] cluster, it functions as an aconitase in cytosol, but loss of its [Fe-S] cluster activates binding to RNA stem loops known as iron-responsive elements (IREs) found in either $5^{\prime}$ or $3^{\prime}$ terminal UTR of numerous gene transcripts, most of which encode proteins implicated in iron metabolism. The translation of proteins encoded by transcripts that contain a $5^{\prime}$ IRE is repressed by binding of IRPs, whereas the stability of mRNAs that contain IREs in the 3' UTR is increased by binding of IRPs. The transcript of aminolevulinate $\delta$, synthase 2 (ALAS2), which catalyzes the first

Conflict of interest: The authors have declared that no conflict of interest exists. Citation for this article: J Clin Invest. 2010;120(5):1749-1761. doi:10.1172/JCI40372. step of heme synthesis in mitochondria of erythroblasts, contains a $5^{\prime}$ IRE, and its translation can be repressed by IRP binding. Mammalian ferrochelatase (FECH) (17) contains a labile [2Fe-2S] cluster that is required for function and stability (18). Thus, activity of the first and last steps of heme biosynthesis in erythroid cells can be governed by iron-sulfur cluster status of cells, either by determining activity of IRP1 or by directly contributing to activity and/or stability of an iron-sulfur enzyme.

Disruption of [Fe-S] biogenesis can cause serious human diseases (19). Although these [Fe-S] synthetic components work at different points in the same pathway, different phenotypes arise from inherited mutations in these genes, including Friedreich ataxia (FRDA) $(20,21)$, ISCU myopathy $(22,23)$, and the recently characterized sideroblastic anemia (SA), associated with a mutation in glutaredoxin 5 (GLRX5) (24). The patient with GLRX5 deficiency and SA has an A294G mutation in the last nucleotide of exon-1, which impedes intron-1 splicing and dramatically reduces GLRX5 mRNA levels. The patient did not develop symptoms until midlife, when he developed microcytic hypochromic anemia, type II diabetes, cirrhosis, and liver iron overload. Inherited SAs comprise a heterogeneous group of genetic disorders characterized by reduced heme synthesis, mitochondrial iron overload, and the presence of ringed sideroblasts (25). Other genetic defects that cause SA include mutations of ALAS2 and ABCB7, both of which are on chromosome X, and the resulting SAs are accordingly referred to as X-linked SAs (XLSA). ABCB7 is an ATP-binding cassette transporter on the inner membrane of mitochondria, which has previously been proposed 

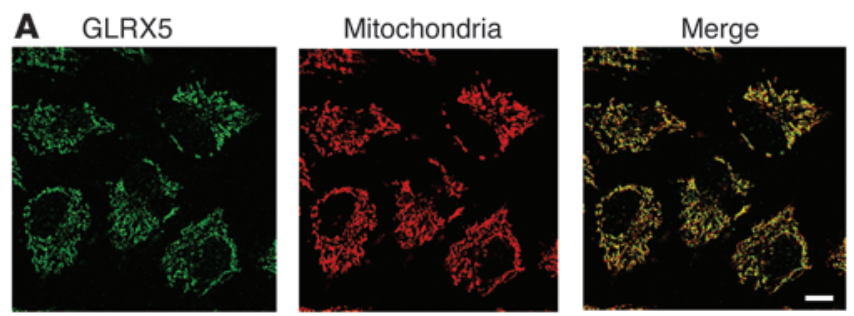

B

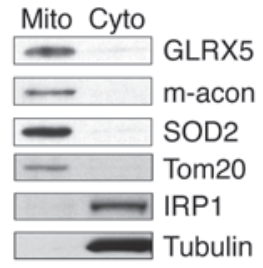

Figure 1

Human GLRX5 localizes to the mitochondrial matrix in HeLa cells. (A) Immunofluorescence staining of endogenous GLRX5 (green) and Tom20 (red) in HeLa cells. Tom20 protein is used as the mitochondrial marker. Merge of the 2 stainings indicates colocalization of GLRX5 and mitochondrial signals. Scale bar: $10 \mu \mathrm{m}$. (B) Western blots of subcellular fractionation of HeLa cells. Mito, mitochondria; cyto, cytosol. Mitochondrial aconitase (m-acon), SOD2, and Tom20 are used as mitochondrial markers. IRP1 (also the cytosolic aconitase) and $\alpha$-tubulin are used as cytosolic markers.

to transport an $[\mathrm{Fe}-\mathrm{S}]$ compound to cytosol $(7,26)$. More recently, a new form of SA was identified, which is caused by mutations of SLC25A38 that may encode an erythroid-specific glycine importer on the mitochondrial inner membrane (27).

Glutaredoxins (GRX) are small redox proteins generally thought to be important for the reduction of oxidized glutathione (GSH) and/or for removal of GSH modifications of proteins. Under oxidative stress, the cysteine thiol groups of many proteins are protected by the reversible addition of the tripeptide, GSH ( $\gamma$-Glu-Cys-Gly), in a process known as glutathionylation, and the removal of GSH requires reduction of the disulfide bond formed between the cysteine of GSH and the cysteine thiol of target proteins. Two major classes of GRX are recognized, including monothiol GRX, which contain a CXXS motif, and dithiol GRX, which contain CXXC domains $(28,29)$. Saccharomyces cerevisiae has 5 GRX, and the phenotypic defects of GRX5 deficiency were suppressed by overexpression of 2 [Fe-S] cluster biosynthetic proteins, SSQ1 or ISA2, but not by the addition of DTT reductant, suggesting that defects of the GRX5 mutant were not due to the alteration of intracellular redox potential (30), but rather to a specific role in biogenesis of [Fe-S] clusters. The human genome contains 4 GRX, including a GRX5 homolog and a GRX3/4-like protein known as PICOT (31). Thus far, there are no known links between PICOT and iron metabolism. Although deficiency of GLRX5 in both zebrafish (32) and a human patient (24) was associated with anemia, the molecular relationship between the anemia and the status of [Fe-S] assembly has not been characterized in GLRX5-deficient mammalian cells.

Here we report that human GLRX5 is essential for mitochondrial [Fe-S] biogenesis. Reconstitution studies reveal that GLRX5 can assemble a [2Fe-2S] cluster, enabling it to function as an [Fe-S] scaffold or sensor. In GLRX5 RNAi cells and GLRX5-deficient patient cells, [Fe-S] synthesis is impaired, and mitochondrial iron overload develops in conjunction with relative cytosolic iron depletion. Transcriptional remodeling in response to cell stress may account for cytosolic iron depletion, which activates IRPs, repressing ALAS2 synthesis in erythroid cells and thus inhibiting heme synthesis in GLRX5 deficiency.

\section{Results}

GLRX5 is a component of mitochondrial [Fe-S] machinery. As it has been previously demonstrated that GRX5 is involved in iron-sulfur cluster biogenesis in yeast and zebrafish $(30,32)$, we hypothesized that a GRX5 human homolog (GLRX5) was required for biogenesis of [Fe-S] clusters in humans. To determine whether GLRX5 was expressed in mitochondria, we performed immunofluorescence staining of endogenous GLRX5 in HeLa cells. In confocal microscopic imaging, GLRX5 expression colocalized with a mitochondrial marker (Figure 1A). By subcellular fractionation and Western blot, GLRX5 was predominantly detected in the mitochondrial fraction (Figure 1B), and we concluded that GLRX5 is a mitochondrial protein.

To study its function, we performed GLRX5 RNAi in HeLa S3 cells. Four siRNA oligos were designed and used to transfect cells. Western blot analysis showed that GLRX5 was reduced to less than $10 \%$ by each oligo (Figure 2A). As both a mitochondrial and a cytosolic form of aconitase, each of which is encoded by a distinct gene, require a [4Fe-4S] cluster for activity (33), we investigated the activity of each isoform in GLRX5-deficient cells. Mitochondrial aconitase enzymatic activity was decreased 2-fold (Figure 2, A and B), while cytosolic aconitase activity was not significantly changed. Since the protein level of aconitases did not change significantly between samples and controls (Figure 2A), the reduction of mitochondrial aconitase activity implied a defect in [Fe-S] cluster assembly. To discern whether there was a subtle effect on [Fe-S] proteins in cytosol, we measured the activity of xanthine oxidase (XO), a [2Fe-2S] enzyme in cytosol. XO activities were all decreased by approximately $30 \%-50 \%$ by GLRX 5 RNAi (Figure 2C). Taken together, GLRX5 knockdown affected the assembly of both [4Fe-4S] and [2Fe-2S] clusters, either in mitochondria or in cytosol. We concluded that GLRX5 is a component of the mitochondrial [Fe-S] synthesis machinery in humans.

Effects of GLRX5 depletion on cellular iron homeostasis. The bifunctional protein IRP1 converts from an active cytosolic aconitase to an IRE-binding protein that regulates intracellular iron metabolism upon loss of its [4Fe-4S] cluster (12). Together with another homolog, IRP2, IRP1 increases expression of the iron uptake protein transferrin receptor 1 (TfR1) and inhibits expression of the iron sequestration protein, ferritin, in cells that are iron depleted. We investigated the status of cellular iron homeostasis in GLRX5depleted cells. The assays showed that total cellular nonheme iron was increased 1.4- to 2.2-fold in GLRX5-deficient cells (Figure 2D), indicating that cellular iron homeostasis was disrupted by GLRX5 depletion. We next studied the subcellular distribution of iron overload, focusing on the mitochondrial and cytosolic fractions, 2 major potential iron repositories in mammalian cells. The total nonheme iron assay on lysate fractions showed that mitochondrial iron of RNAi samples was increased 2.3-fold over control levels, whereas cytosolic iron levels were not statistically different between RNAi samples and controls (Figure 2E). These results indicated that most of the accumulated iron was stored in mitochondria.

The iron status in cytosol was further examined by Western blotting of IRP2 and H-ferritin. IRP2 undergoes iron-dependent degradation in iron-replete cells, whereas IRP2 levels increase in cells that are depleted of cytosolic iron (12). In contrast, expression 
A

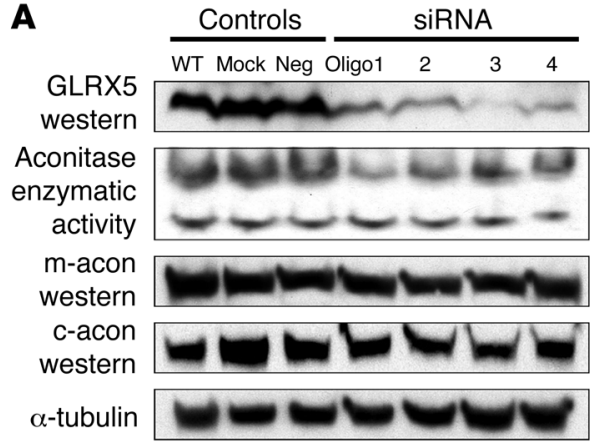

B

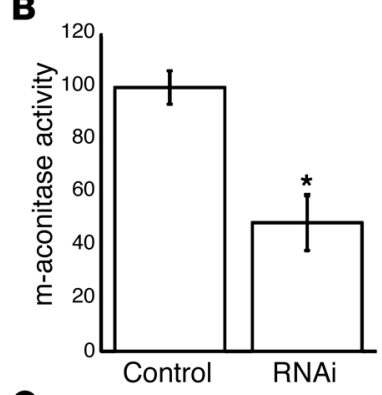

C
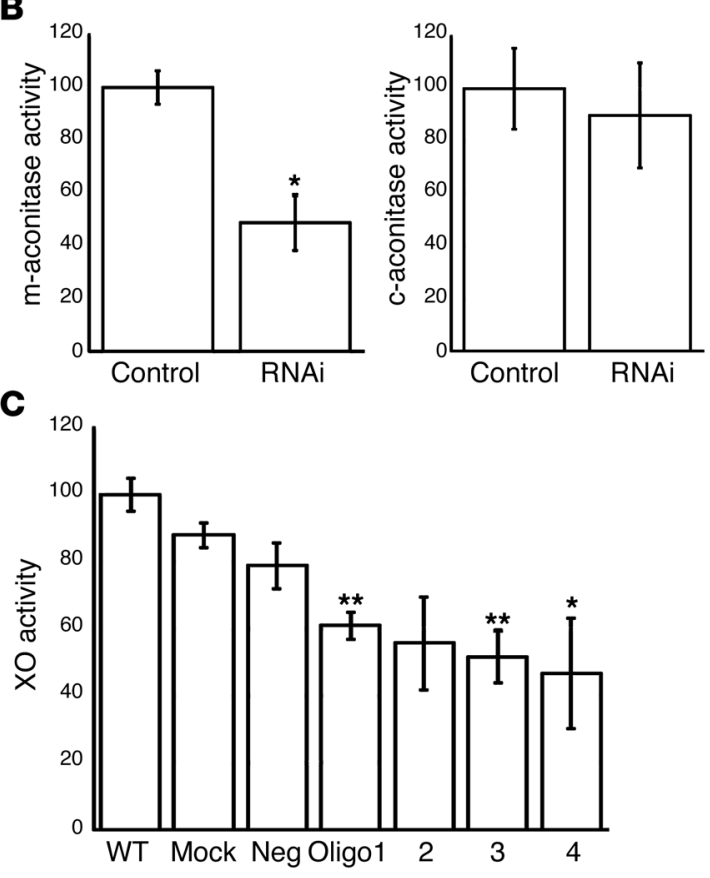

D

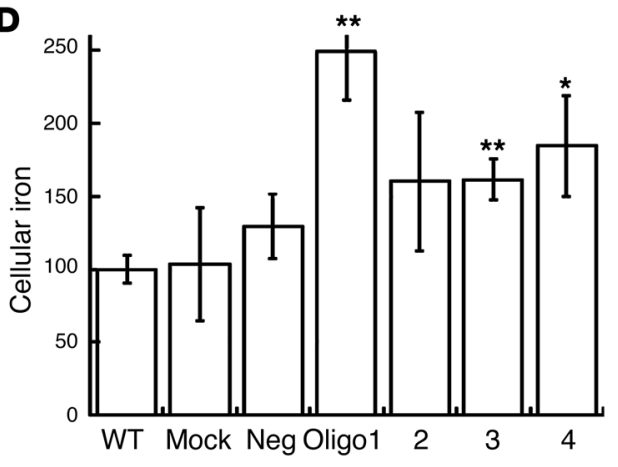

E
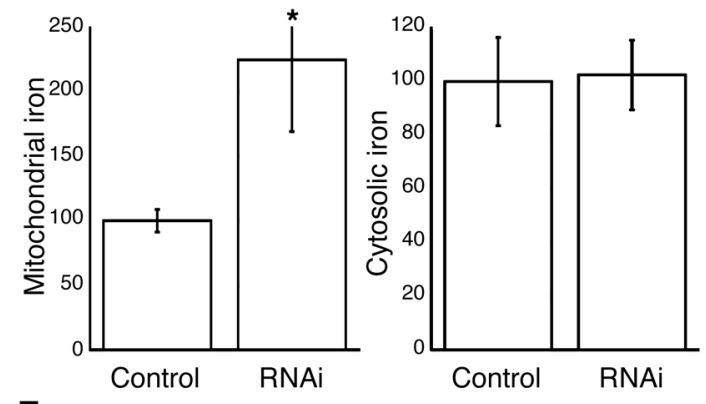

F

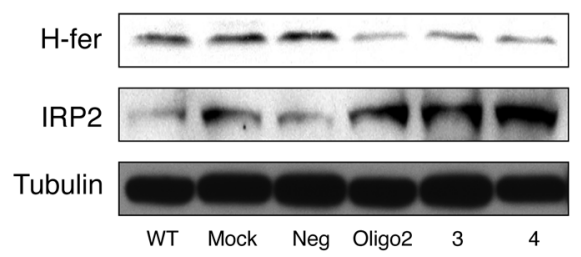

G

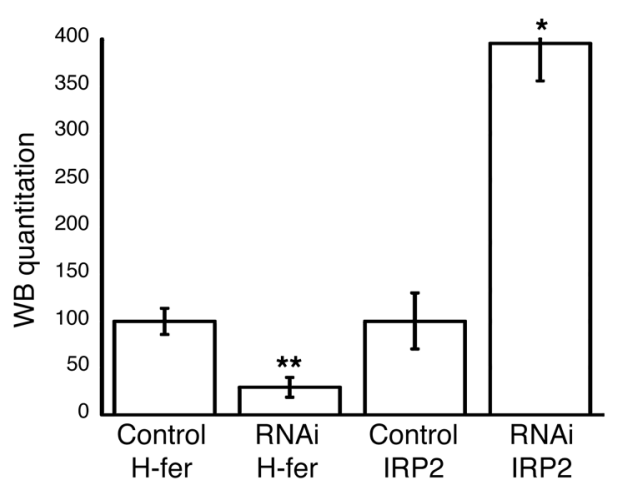

Figure 2

Knockdown of GLRX5 by siRNA. (A) GLRX5 in HeLa S3 cells was knocked down using 4 siRNA oligos (1 to 4). WT, mock, and neg. are negative controls (mock: same treatment as RNAi but without any oligo). The in-gel aconitase assay shows mitochondrial aconitase (m-acon) activity (top band) and cytosolic aconitase (c-acon) activity. Western blots of m-acon, c-acon, and $\alpha$-tubulin are shown. (B) Quantitation of band intensity of aconitase activity in part A. $P=0.015$ for RNAi m-acon. ${ }^{*} P<0.05$; ${ }^{* \star} P<0.01$. m-aconitase activity (percentage of control). (C) XO activity assay. Results are expressed as a fraction of WT (same for the following quantitation). For treatments by oligo1-4, $P=0.01,0.07,0.007$, and 0.027 , respectively. XO activity (percentage of WT control). (D) Cellular nonheme iron quantitation. For treatments by ologo $1-4, P=0.005,0.2,0.01$, and 0.04, respectively. Cellular iron (percentage of WT control). (E) Nonheme iron quantitation with subcellular fractionation. Control, control cells (WT, mock, and neg.); RNAi, siRNA-treated cells (by oligo1-4); mito, mitochondria fraction; cyto, cytosol fraction. For sample of RNAi-mito, $P=0.03$. Mitochondrial iron (percentage of control). (F) Western blot for IRP2 and $\mathrm{H}$-ferritin (H-fer). The $\alpha$-tubulin is loading control. (G) Quantitation of band intensity of Western blot in panel F. $P=0.0003$ and 0.021 , respectively. WB quantitation (percentage of control $\mathrm{H}$-ferritin). 
A

HsGRX5 MmGRX5 DrGRX5 ScGRX5 AtGrxS14 AtGrxS16

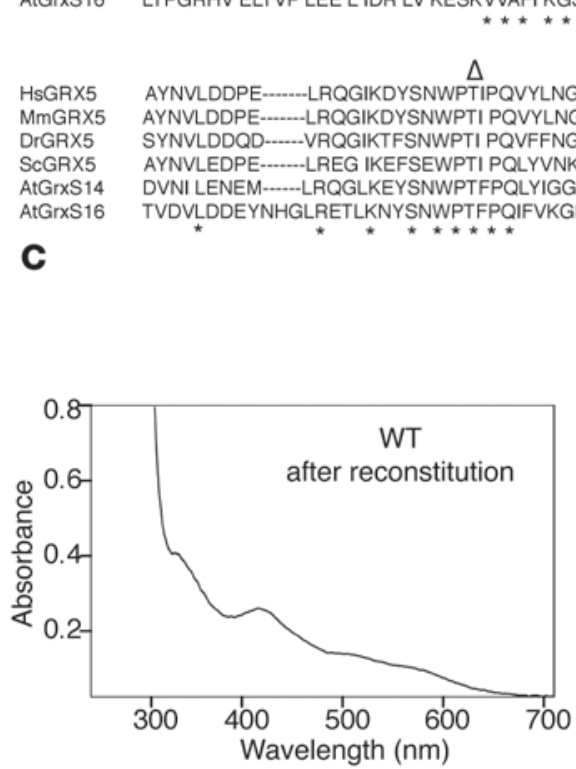

$\mathbf{F}$

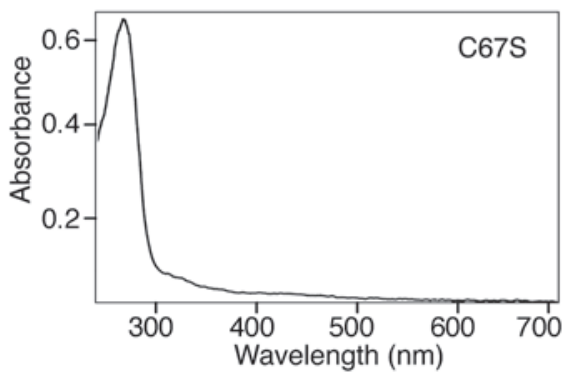

$\Delta$ $\Delta$

VRAAGSGAGGGGSAEQLDALVKKDKVVVFLKGTPEQPQCGFSNAVVQI LRLHGVR-...DYA 86 VRAASS--..... GGQAEQLDALVKKDKVVVFLKGTPEQPQCGFSNAVVQI LRLHGVR--.-DYA 82 ARLMCS........SAGQKNLEEMVKKDKVVVFMKGTPAQPMCGFSNAVVQILRMHGVD...-NYA 86 LRYQNR MYLSTE IRKA IEDA I ESAPVV LFMKGTPEFPKCGFSRAT IGL LGNQGVDPAKFA 81 KFRCSASALTPOL KDTLEKL VNSEKVLFMKGTRDFPMCGFSNTVVILKNLNVPITPGRHV ELTVP LEE LIDR LV KESKVVAFI KGSR SAPQCGFSQRVVGILES QGVD-...-YE 237

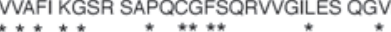

\section{$\Delta$}

AYNVLDDPE-..---.-LRQGIKDYSNWPTIPQVYLNGEFVGGCDILLQMHQNGDLVEELKKLGI 143 AYNVLDDPE-...-LRQGIKDYSNWPTI PQVYLNGEFVGGCDILLQMHQNGDLVEELKKLGI 139

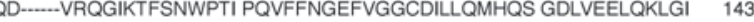
作 作 TVDVLDDEYNHGLRETLKNYSNWPTFPQIFVKGELV GGCDILTSMYEN GELANILN-.----- 293

$$
{ }^{*}{ }^{* * * *} \underset{* *}{ }
$$

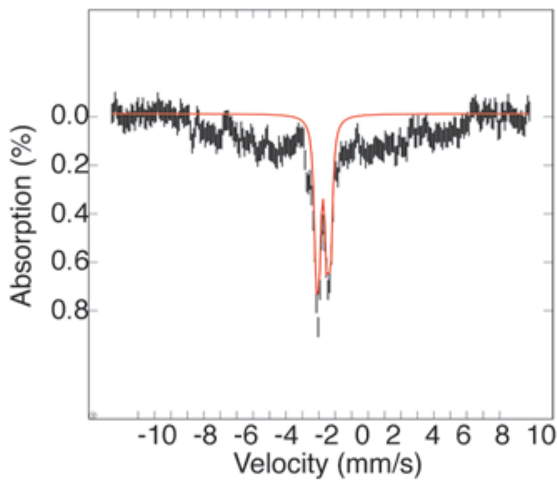

G

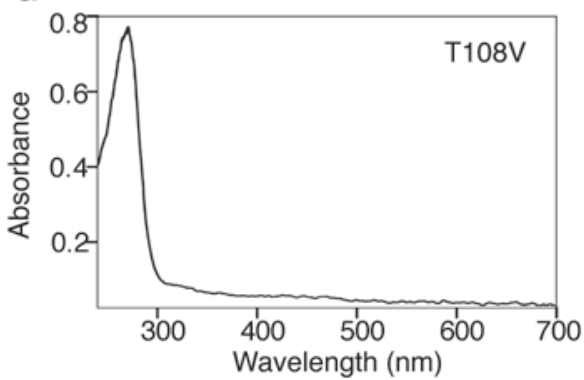

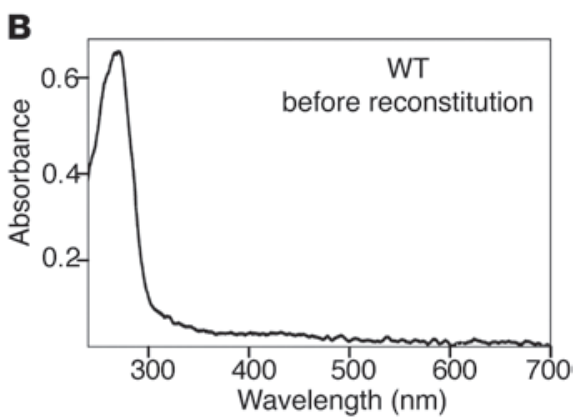

$\mathbf{E}$

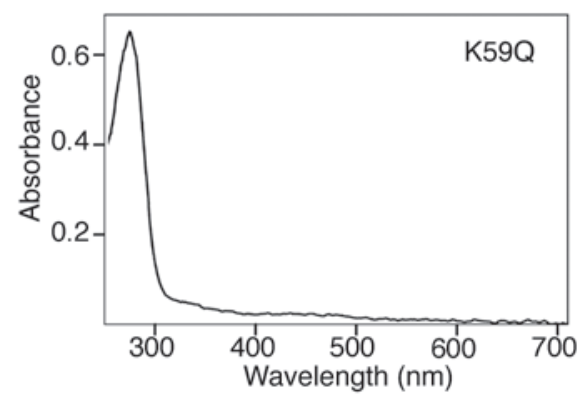

H

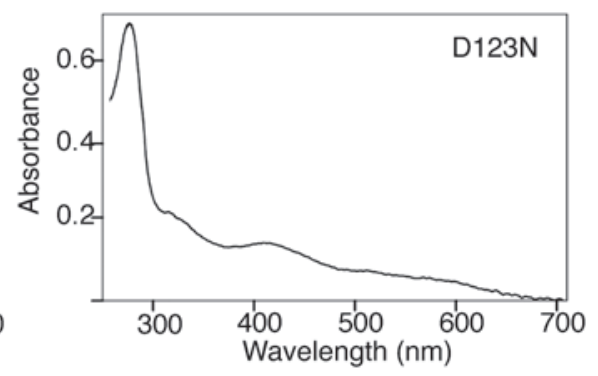

Figure 3

A [2Fe-2S] cluster can be reconstituted in GLRX5 in vitro. (A) Protein sequence alignment of eukaryotic GLRX5 homologs. The nonconserved $\mathrm{N}$ - and $\mathrm{C}$-terminal sequence is not shown. Identical residues are marked by asterisks, whereas residues that are mutagenized in subsequent panels are labeled with triangles. Hs, Homo sapiens; Mm, Mus musculus; Dr, Danio rerio; Sc, Saccharomyces cerevisiae; At, Arabidopsis thaliana. (B) UV-visible spectrophotometry of purified WT GLRX5 before anaerobic [Fe-S] reconstitution. (C) UV-vis spectrophotometry of purified WT GLRX5 after anaerobic reconstitution. The reconstitution was performed as described in Methods using reduced GSH. (D) Mossbauer analysis of reconstituted WT GLRX5 protein. The data (hatched marks) were recorded at $4.2 \mathrm{~K}$ in a parallel applied field of $50 \mathrm{mT}$. The solid line (red) is a theoretical estimate of parameters associated with [2Fe-2S $]^{2+}$ clusters using 2 equal intensity quadrupole doublets (35\%) with parameters $\delta=0.25$, $\Delta \mathrm{E}_{\mathrm{Q}}=0.47, \Gamma=0.28 / 0.28$, and $\delta=0.27, \Delta \mathrm{E}_{\mathrm{Q}}=0.9$, and $\Gamma=0.28 / 0.28$. (E) UV-vis spectrophotometry of $\mathrm{K} 59 \mathrm{Q}$ mutant $\mathrm{GLRX}$ after reconstitution. The substitution was introduced by site-directed mutagenesis. (F) UV-vis spectrophotometry of C67S mutant GLRX5 after reconstitution. (G) UV-vis spectrophotometry of T108V mutant GLRX5 after reconstitution. (H) UV-vis spectrophotometry of D123N mutant GLRX5 after reconstitution.

of $\mathrm{H}$-ferritin, the iron storage protein in cytosol, increases in iron replete and decreases in iron depleted cells because of translational regulation by IRP proteins. We observed that IRP2 was increased 4-fold in GLRX5-depleted cells, whereas H-ferritin was decreased to $30 \%$ of control levels (Figure 2, F and G), suggesting that the cytosol was relatively iron deficient compared with controls. These results suggested that GLRX5 depletion causes mitochondrial iron overload in conjunction with relative cytosolic iron deficiency and that GLRX5 is important to cellular iron homeostasis.

GLRX5 functions as a potential [Fe-S] scaffold or sensor. Recently, a number of GRX from various species have been recognized as potential [Fe-S] proteins (34-37). Most of the GLRX5 proteins from mammals and other vertebrates contain the motif CGFS and are monothiol GRX in which an iron-sulfur cluster bridges 2 subunits, using the cysteine from the CGFS motif of each monomer as a ligand $(34,35)$ (Figure $3 \mathrm{~A})$. To determine whether human GLRX5 can bind an [Fe-S] cluster, we performed in vitro [Fe-S] cluster reconstitution with recombinant GLRX5. Before reconstitution, the purified recombinant protein was colorless and showed only the A280 absorption peak that reflects protein in UV-vis spectrophotometry (Figure 3B). However, after reconstitution and subsequent purification, the protein acquired a dark brown color and displayed additional strong absorption peaks at 330 and $420 \mathrm{~nm}$, with a little shoulder at $520 \mathrm{~nm}$ (Figure 3C), an absorption pattern 
A

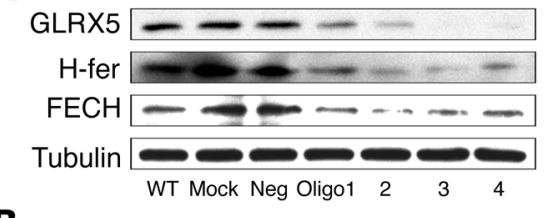

B

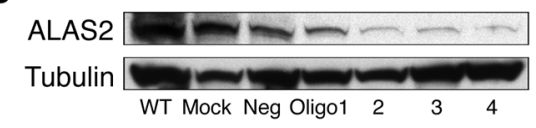

C

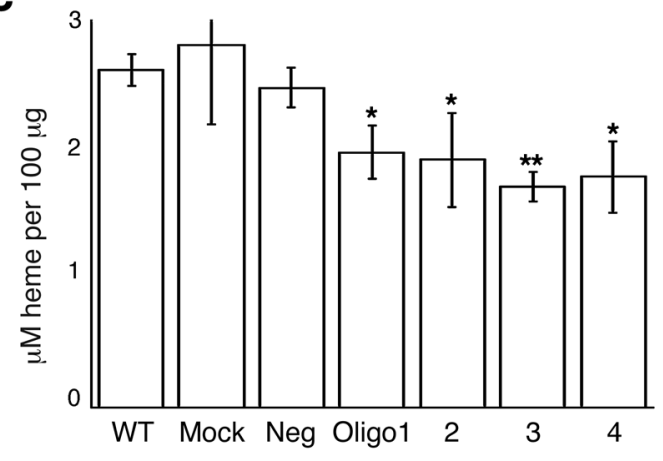

that is typical of [2Fe-2S] clusters. Notably, the presence of reduced GSH was required for [Fe-S] assembly in GLRX5. Mossbauer analysis (Figure 3D) of the reconstituted GLRX5 further supported the presence of a $[2 \mathrm{Fe}-2 \mathrm{~S}]^{2+}$ cluster. The signature of Mossbauer spectrum can be simulated with doublets having parameters that are consistent with a $[2 \mathrm{Fe}-2 \mathrm{~S}]^{2+}$ cluster.

In GRX that have been reconstituted thus far, a catalytic cysteine residue from each monomer is required to ligate the [Fe-S] cluster, along with $2 \mathrm{GSH}$ molecules, and several conserved residues are thought to be involved in docking of the GSH ligand to the protein (35). We next investigated the involvement of several conserved residues, K59, C67 (in the CGFS active domain), T108, and D123, by site-directed mutagenesis and subsequent reconstitution (Figure 3A). Mutagenesis of residues K59Q, C67S, and $\mathrm{T} 108 \mathrm{~V}$ abolished the characteristic [Fe-S] absorbance pattern after reconstitution (Figure 3, E-G), indicating that the assembly of an [Fe-S] cluster in human GLRX5 requires these residues, likely for docking of the GSH ligand. These data suggest that human GLRX5 may function as a potential [Fe-S] scaffold, similar to the plant GRX, AtGrxS14, and AtGrxS16 (Figure 3A), which are proposed to function as scaffold proteins (34).

A potential additional role of GLRX5 in erythropoiesis. The expression profile of a gene in various tissues can give potential clues about function, and a bioinformatics analysis showed that human GLRX5 is minimally expressed in all tissues, but is highly expressed in CD71 early erythroid cells of bone marrow (data not shown). The expression pattern of GLRX5 is very similar to those of human ALAS2 and FECH, which are involved in heme biosynthesis in erythroid tissues. To experimentally verify the tissue-specific expression of mammalian GLRX5, we performed in situ hybridization of GLRX5 in adult mouse tissues. GLRX5 transcripts were expressed at low levels in liver, kidney, lung, heart, testis, and skeletal muscle, consistent with previously reported results (ref. 34 and data not shown). In contrast, GLRX5 was abundantly expressed in bone marrow cells and some parts of brain including the hippocampus and cerebellum (particularly in Purkinje cells, granule cells, and deep cerebel-

\section{Figure 4}

Knockdown of GLRX5 in K562 cells by siRNA reveals that GLRX5 is specifically required for heme synthesis in erythroblasts. (A) GLRX5 in K562 cells was knocked down by 4 siRNA oligos (1 to 4), respectively. WT, mock, and neg are negative controls. $\alpha$-tubulin is loading control for the Western blots. (B) Western blot of ALAS2. $\alpha$-tubulin is loading control. (C) Cellular heme quantitation. $y$ axis shows the unit of heme concentration $(\mu \mathrm{M} / 100 \mu \mathrm{g}$ lysate). For treatments by ologo $1-4, P=0.018$, $0.04,0.001$, and 0.027 , respectively. ${ }^{\star} P<0.05$; ${ }^{\star \star} P<0.01$. Results represent averages of 3 replicates, and error bars are indicated.

lar nuclei) (Supplemental Figure 1; supplemental material available online with this article; doi:10.1172/JCI40372DS1). Further analysis of GLRX5 transcripts by quantitative RT-PCR (qRT-PCR) with sorted cells from mouse bone marrow revealed that GLRX5 was at least 100-fold more expressed in erythroblast cells than in macrophages and any other bone marrow cells (Supplemental Figure 2A). The expression analysis implied that GLRX5 may have an important role in erythropoiesis and also in cerebellar neuronal function. The markedly high expression of GLRX5 in erythroblasts suggests that the promoter of this gene could be selectively activated in erythroid lineage cells during erythropoiesis, and we have observed that GLRX5 levels increase in differentiating MEL cells, a mouse erythroid cell line (Supplemental Figure 2, B-D).

To determine whether GLRX5 is required for heme synthesis and erythropoiesis, we performed GLRX5 knockdown experiments in an erythroid K562 cell line. GLRX5 was reduced to less than $10 \%$ by siRNA oligo treatment (Figure 4A). H-ferritin expression was also markedly decreased, consistent with IRP-mediated translational repression (Figure 4A). Under iron depletion, ALAS2 expression could be potentially inhibited by IRPs, as was demonstrated in GLRX5knockout zebrafish (32), because it contains an IRE in its $5^{\prime}$ UTR. Indeed, ALAS2 levels were significantly decreased in GLRX5-depleted K562 cells (Figure 4B). Unexpectedly, protein levels of another heme synthesis protein, $\mathrm{FECH}$, were also decreased by GLRX5 knockdown (Figure 4A). Both ALAS2 and FECH are important enzymes in heme biosynthesis in erythroid tissues (17), and heme content was accordingly decreased 25\%-36\% in siRNA-treated K562 cells (Figure 4C). In contrast, neither FECH protein levels nor heme content were decreased in siRNA-treated HeLa S3 cells (Supplemental Figure 3). Therefore, the effect of GLRX5 depletion on FECH protein and heme synthesis appeared to be specific for erythroid cells. Taken together, these data indicated that in addition to a general function in [Fe-S] synthesis and cellular iron homeostasis (Figure 2), the human GLRX5 has an important role in erythropoiesis.

Disruption of cellular iron homeostasis in an SA patient with a GLRX5 mutation. Recently, a patient with SA and a mutation that caused abnormal splicing of GLRX5 was identified in Italy (24). To better understand the pathogenesis of this GLRX5-associated SA, we studied iron homeostasis in a lymphoblast cell line and fibroblasts derived from the patient. As shown by Western blot, we established that GLRX5 protein was undetectable in patient lymphoblasts and fibroblasts (Figure 5, A and B). Since GLRX5 is a component of [Fe-S] synthesis machinery (Figure 2), we measured the activity of mitochondrial and cytosolic aconitases. Mitochondrial aconitase activity was undetectable (Figure 5C), and cytosolic aconitase activity was decreased to less than 10\% (Figure 5C), even though protein levels of IRP1 did not change (Figure 5D). As expected, absence of the [Fe-S] cluster of IRP1 resulted in markedly increased IRE-binding activity (Figure 5D). To assess the status of other [Fe-S] proteins, we evalu- 
A

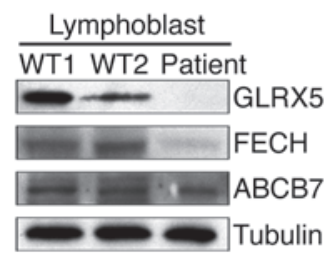

B

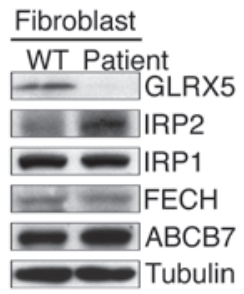

C

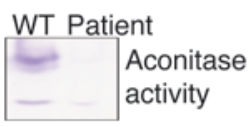

D

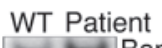

Bund shift

- IRP1 WB

Tubulin WB
E

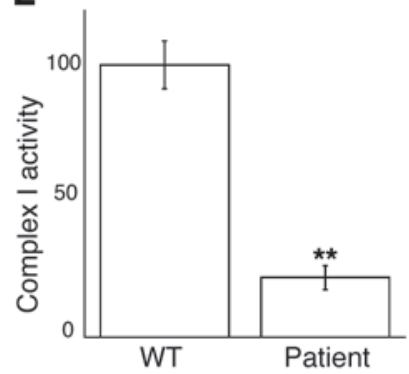

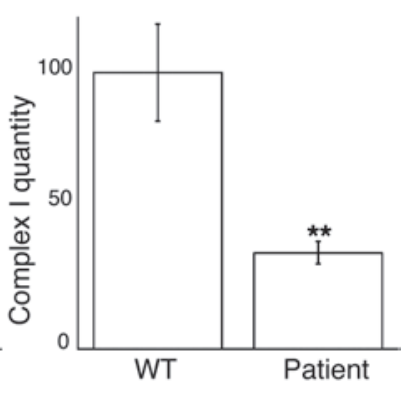

$\mathbf{F}$

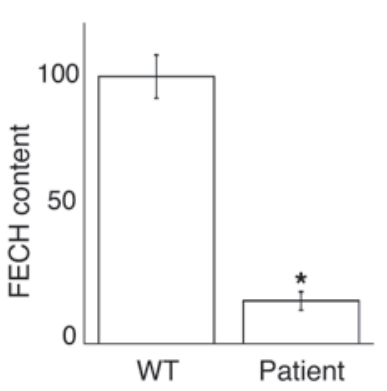

G

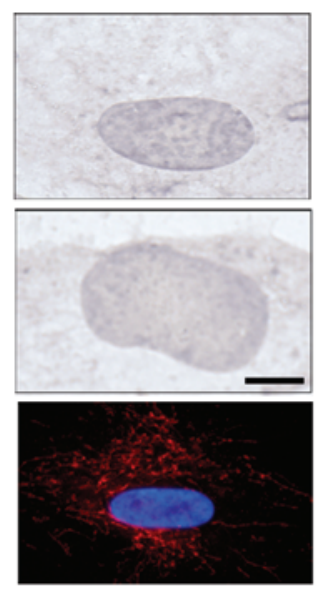

Patient

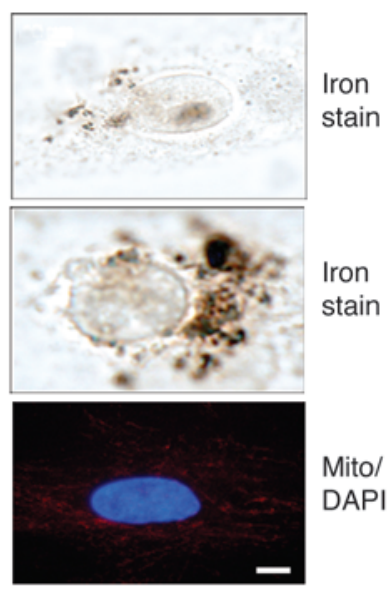

H

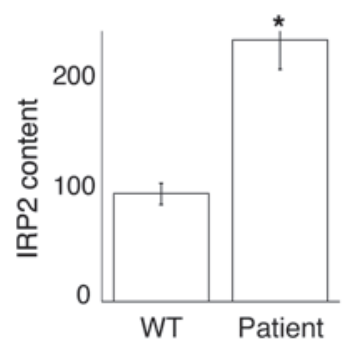

\section{Figure 5}

In cells from a GLRX5-deficient patient, aconitase activities are low and IRE-binding activities are increased. (A) Western blot analysis of patient lymphoblast cells. Lymphoblast 1 and 2 are WT controls. ABCB7 (ATP-binding cassette, sub-family B, member 7) is a control for mitochondrial proteins. $\alpha$-Tubulin is loading control. (B) Western blot analysis of patient fibroblast cells. (C) In-gel aconitase activity assay with patient fibroblast and control cells. The top band is mitochondrial aconitase, whereas the bottom is cytosolic aconitase. (D) Band shift assay with patient fibroblast and control cells. IRP1 and tubulin Western blots verified equal loading. (E) Complex I activity assay and quantitation with patient fibroblast and control cells. Values for WT control cells are set at 100. Results represent the average of 3 repeats. For activity and quantitation, $P=0.00018$ and 0.0032 , respectively. (F) Quantitation of band intensity of FECH in A. Value for WT control is set at 100. Results represent the average of 3 repeats. $P=0.021$. (G) Perls DAB nonheme iron staining in patient fibroblast cells. The bottom panel shows immunofluorescence staining of mitochondria using Tom20 (red) as a mitochondrial marker of WT and patient fibroblasts. Scale bars: $10 \mu \mathrm{m}$. (H) Quantitation of band intensity of IRP2 in B. Value for WT control is set at 100. Results represent the average of 3 repeats. $P=0.03$. ${ }^{*} P<0.05 ;{ }^{* *} P<0.01$.

ated complex I, a major multimeric complex on the inner mitochondrial membrane that contains $2[2 \mathrm{Fe}-2 \mathrm{~S}]$ and $6[4 \mathrm{Fe}-4 \mathrm{~S}]$ clusters that relay electrons through the complex (38). Complex I activities were decreased to $20 \%$ of normal, whereas quantities of complex I protein were diminished less notably to approximately $40 \%$ of normal in patient fibroblasts (Figure 5E). These results indicated that [Fe-S] cluster biogenesis was severely impaired in the patient, with loss of aconitase activity, decrease of complex I activity, and activation of the IRE-binding activity of IRP1 (cytosolic aconitase) in patient fibroblasts, as indicated by band shift assay (Figure 5D).

Since the mammalian heme synthesis protein, $\mathrm{FECH}$, contains a [2Fe-2S] cluster (39), we tested to determine whether FECH levels were affected in the GLRX5-deficient patient cells. By Western blot, FECH protein levels were not changed in fibroblasts of the patient versus control (Figure 5B). In contrast, FECH levels in the patient lymphoblast cell line were decreased to $16 \%$ of WT control (Figure 5, $A$ and $F$ ), whereas levels of the inner mitochondrial membrane protein, ABCB7, were not decreased in patient cells. These results implied that the reduction in FECH levels of patient lymphoblast cells was not associated with reductions in other mitochondrial proteins. We had previously demonstrated that $\mathrm{FECH}$ protein was reduced in GLRX5-depleted K562 erythroid cells (Figure 4) but not in HeLa S3 epithelial cells (data not shown). As both lymphoblast and erythroid cells are hematopoietic tissues, these results are consistent with the possibility that GLRX5 has a specific role in the heme biosynthetic pathway of hematopoietic cells, perhaps functioning as a specific [2Fe-2S] donor to FECH.

[Fe-S] biogenesis and heme biosynthesis are among the most important pathways of cellular iron metabolism (40), and we have observed that the machineries of both processes are impaired in the patient. We further analyzed the cellular iron distribution in patient cells using the Perls DAB iron stain, and we identified punctate iron deposits in a pattern consistent with mitochondrial iron overload in fibroblasts derived from the patient (Figure 5G), whereas iron staining was diffuse in cytosol and nuclei of WT fibroblasts. The intensity of mitochondrial staining identified with the mitochondrial marker Tom 20 was somewhat attenuated in fibroblasts from the patient, perhaps indicating some mitochondrial compromise (Figure 5G). To more directly evaluate cytosolic iron status, we measured IRP2 levels as an indicator of cytosolic 
A
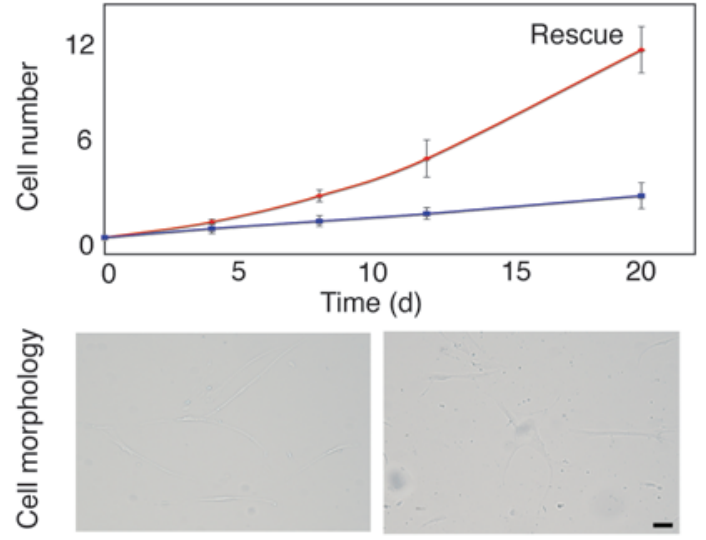

GLRX5 rescue

C

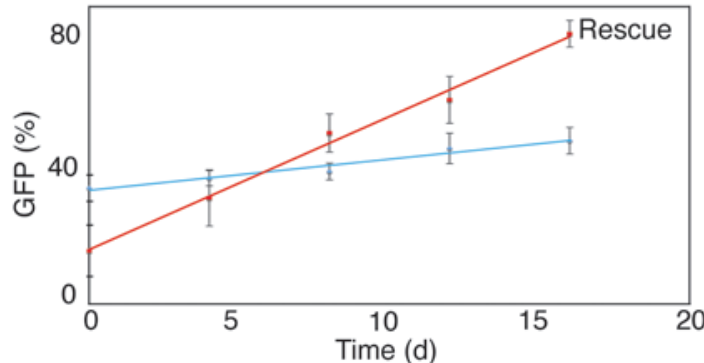

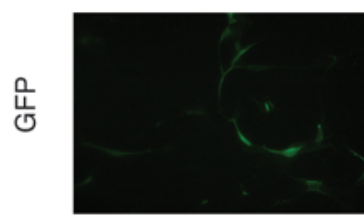

GLRX5 rescue

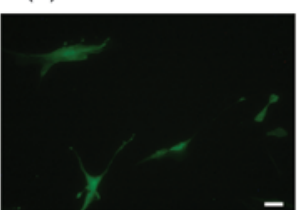

Ctl
B
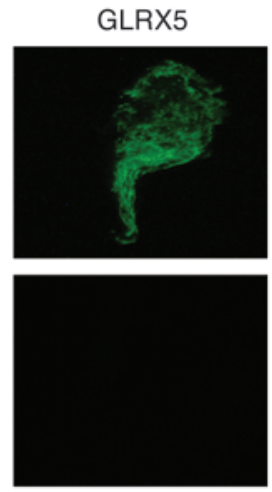

D
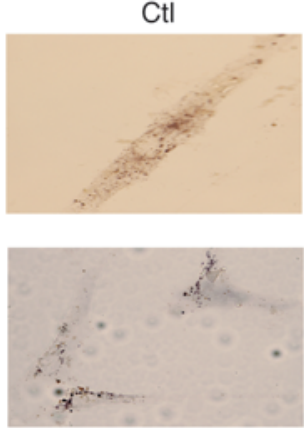

E

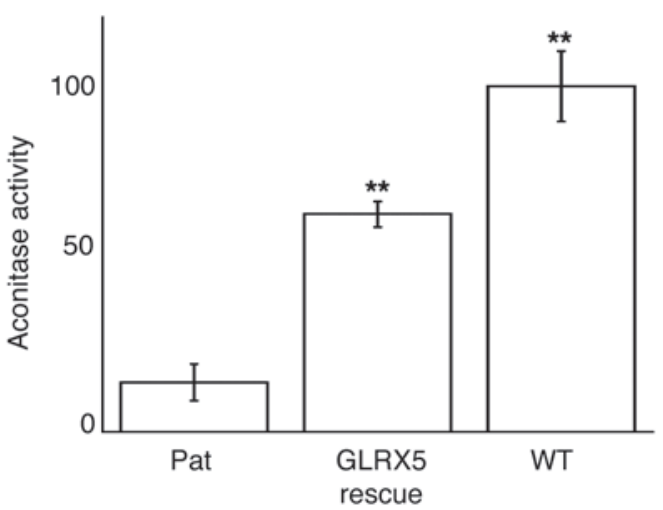

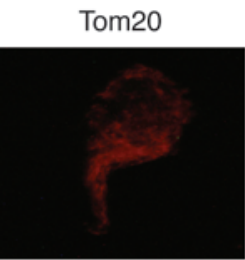
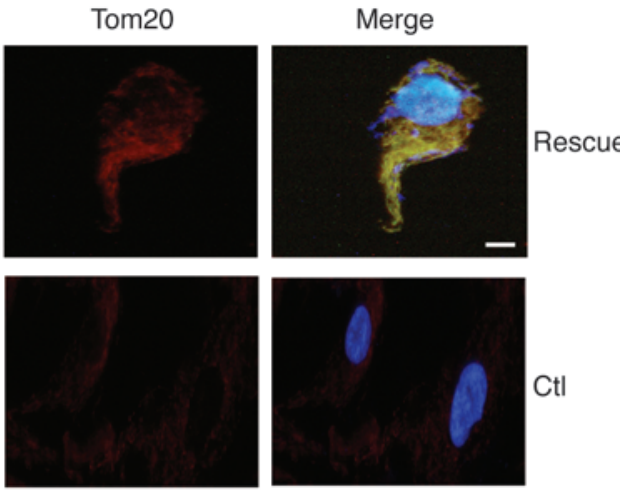

GLRX5 rescue
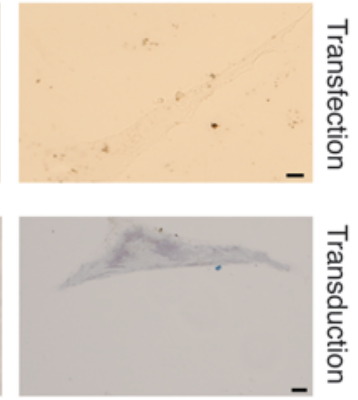

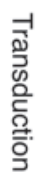

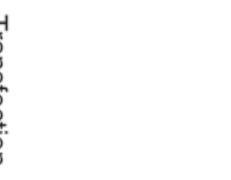

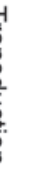
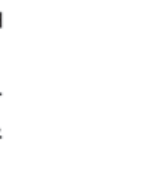

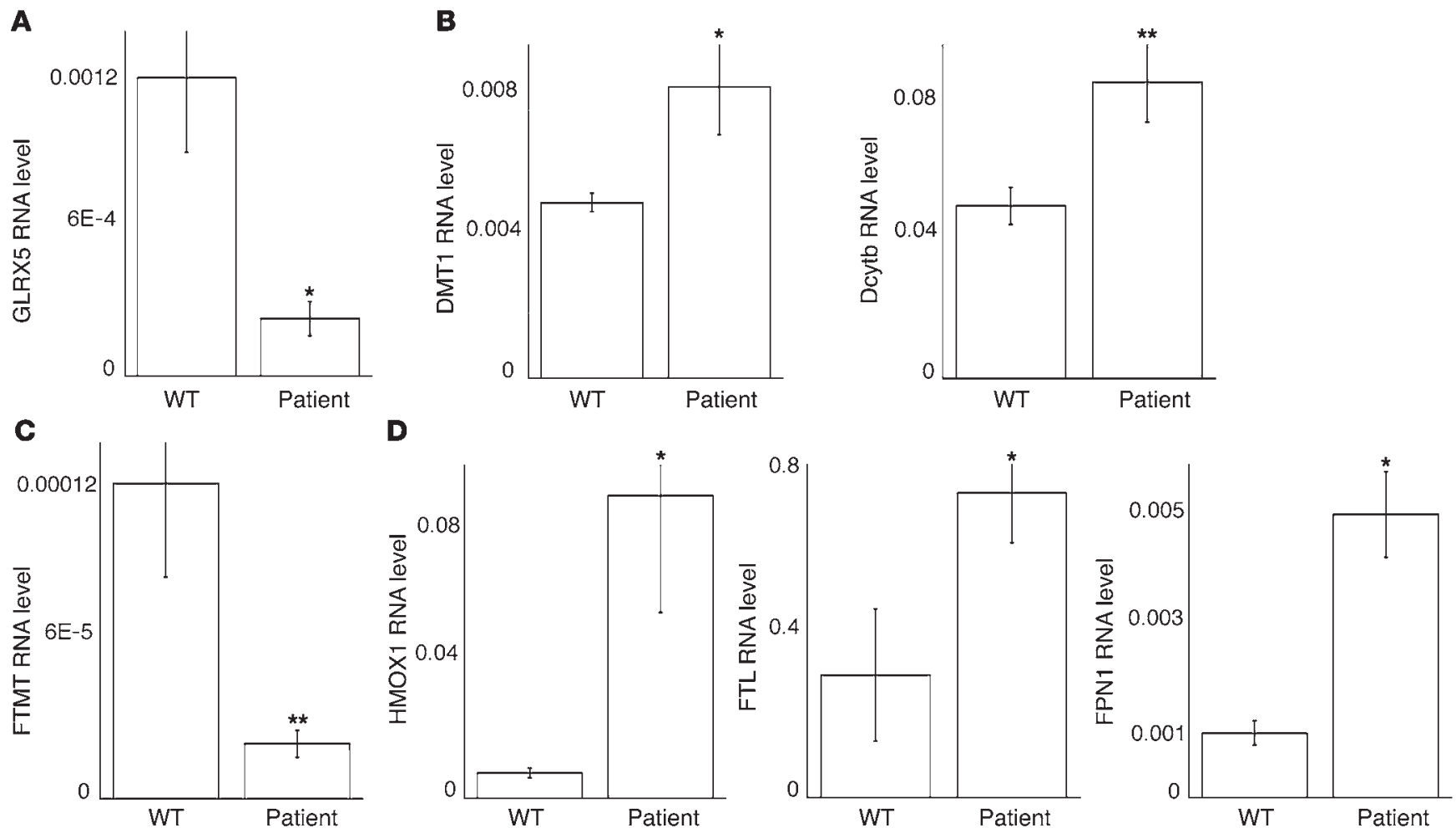

Figure 7

Transcriptional remodeling of iron metabolism genes in patient fibroblasts reveals evidence for cell stress and role of ferroportin in a cellular stress response. (A) qRT-PCR analysis of GLRX5 transcripts. A WT human fibroblast cell line is used as control. Results are verified by the transcript level of actin and represent the average of 3 repeats; the same is true for the following results. $P=0.02$. (B) qRT-PCR analysis of DMT1 and Dcytb transcripts. $P=0.015$ and 0.009 , respectively. (C) qRT-PCR analysis of FTMT transcripts. $P=0.0086$. (D) qRT-PCR analysis of HMOX1, FTL, and FPN1 transcripts. $P=0.015,0.048$, and 0.02 , respectively. ${ }^{*} P<0.05 ;{ }^{\star \star} P<0.01$.

ing plasmid (pCMV-GLRX5 as described in Methods), the cell growth rate increased significantly (Figure 6A), and the doubling time decreased to 4-5 days from 8-10 days for control cells. Additionally, cell morphology changed from thick cell bodies with few extensions to long, thin cells with numerous extensions (Figure 6A). The expression of GLRX5 protein was confirmed by immunofluorescence experiments, in which control patient cells lacked any GLRX5 signal, while the rescued patient cells demonstrated significant GLRX5 fluorescence (Figure 6B). The GLRX5 fluorescence colocalized with Tom20, a mitochondrial marker. Furthermore, Tom 20 fluorescence was overall stronger in rescued cells, suggesting that the presence of GLRX5 improved mitochondrial morphology. Further, rescue experiments were also performed using viral transduction methods. A WT gene sequence of human GLRX5 was inserted into an HIV1 lentiviral vector (41) upstream of a GFP open reading frame, and an internal ribosome entry site (IRES) sequence in between the 2 open reading frames allowed translation of these 2 proteins separately in host cells. The occurrence of GFP fluorescence in transduced cells indicated that GLRX5 was also expressed (Figure 6C), but GFP fluorescence was weaker in cells rescued with the GLRX5-GFP compared with GFP alone, probably because the translation of GFP driven by the IRES was less efficient than the translation driven by the CMV promoter. Likely for the same reason, we counted a lower GFP percentage (ratio of GFP expressed cell number to total cell number) for GLRX5-rescued cells on day 0 (Figure 6C), although they were expected to be similarly transduced because of the transduction efficiency of the lentiviral vector. From day 0 to day 16, the GFP percentage was always around $40 \%$ in control cells transduced with GFP alone (Figure 6C). In contrast, the percentage of GFPpositive cells increased from $16 \%$ on day 0 to $82 \%$ on day 16 in GLRX5-rescued cells. These results indicated that the rescued cells were gaining a growth advantage over nonrescued counterparts, and they also suggested that GLRX5 is required for normal cell growth. In addition, the GLRX5-rescued cells had long graceful extensions, whereas cell bodies of nonrescued cells were wide and processes were attenuated (Figure 6E), consistent with results observed in transfection experiments (Figure 6A). Next, we investigated the mitochondrial iron overload, a hallmark of the GLRX5 deficiency phenotype. Similar to the original patient cells, the control cells transfected with pCMV alone or transduced with GFP alone demonstrated profound punctate iron deposits, suggesting mitochondrial iron accumulation (Figure 6D). In contrast, rescue of patient cells resulted in loss of mitochondrial iron overload, as confirmed by both transfection and transduction experiments. Finally, we measured cellular aconitase activity, which contains $[\mathrm{Fe}-\mathrm{S}]$ clusters. The aconitase activity of patient fibroblasts was restored to over $60 \%$ of WT cells by GLRX5 viral transduction (Figure 6E). The success of rescuing GLRX5-deficient patient cells using lentivirus in this study suggests that gene therapy could be a promising treatment for other GLRX5-deficient patients that may be diagnosed in the future. 
A
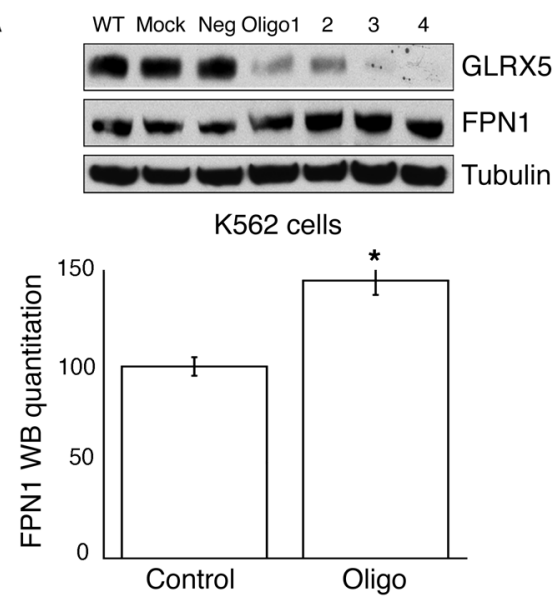

B
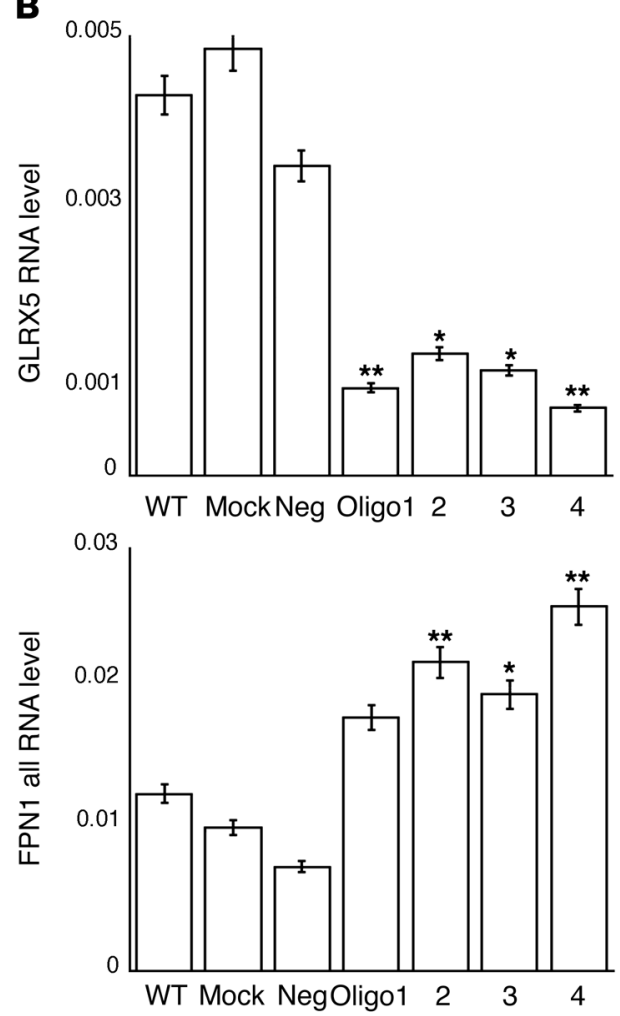

WT Mock Neg Oligo1 $2 \quad 3 \quad 4$

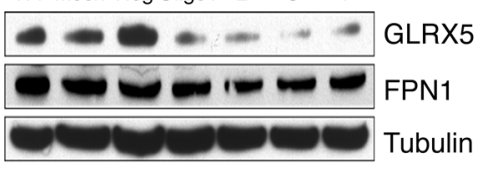

HeLaS3 cells
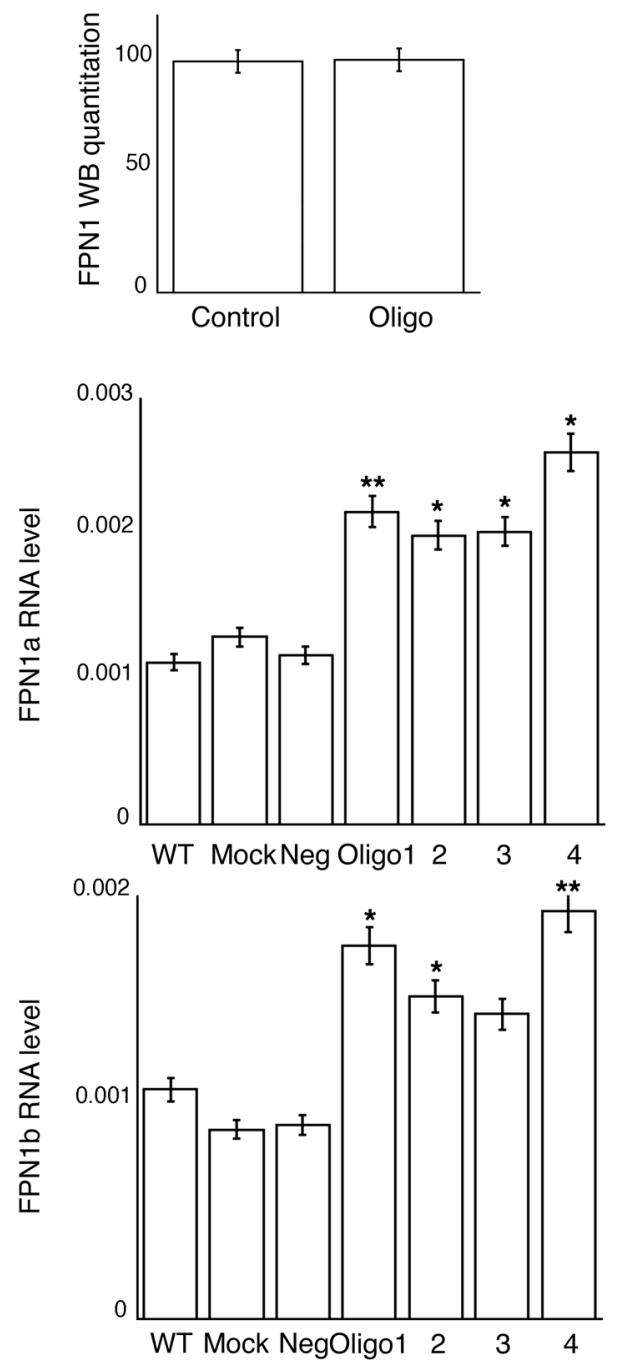

\section{Figure 8}

Ferroportin protein levels are increased by GLRX5 depletion only in erythroid precursor cells. (A) Western blots of FPN1 in GLRX5 siRNA-treated K562 and HeLa S3 cells, in which GLRX5 was knocked down by 4 siRNA oligos (1 to 4). WT, mock, and neg are negative controls. FPN1, ferroportin. $\alpha$-Tubulin is loading control. (B) qRT-PCR analysis of GLRX5, FPN1a, FPN1b, and total FPN1 transcripts in GLRX5 siRNA-treated K562 cells. Results were verified by the transcript level of actin, and represent the average of 3 repeats. $P=0.0063$, $0.011,0.015$, and 0.007 for GLRX5 in oligo $1-4 ; P=0.0076,0.04,0.025$, and 0.017 for FPN1a in oligo 1-4; $P=0.013,0.034,0.12$, and 0.004 for FPN1b in oligo $1-4$; and $P=0.138$, $0.0087,0.021$, and 0.0053 for FPN1 total in oligo $1-4 .{ }^{*} P<0.05$; ${ }^{\star \star} P<0.01$.
Transcriptional remodeling in patient fibroblast cells. To understand the phenotype of mitochondrial iron overload and cytosolic iron deficiency in fibroblast cells derived from the patient, we performed a microarray analysis (Supplemental Table 1) and confirmed many of the changes of iron metabolism genes by qRT-PCR. Total GLRX5 transcripts were decreased 6-fold in patient fibroblasts (Figure 7A). Since the gene mutation interferes with normal splicing (24), the decrease of GLRX5 mRNA suggested that unspliced GLRX5 transcripts were largely degraded. We analyzed expression of cellular iron import proteins, including TfR1, and divalent metal transporter 1 (DMT1) and duodenal cytochrome B $(D c y t b)$ genes. TfR1 transcripts were not significantly changed (not shown), whereas DMT1 and Dcytb transcripts in patient cells were increased 1.7-fold and 1.8-fold, respectively (Figure 7B), which could support increased iron import into cells. To attempt to understand the phenomenon of mitochondrial iron overload that is observed in cells with compromised [Fe-S] biogenesis, we evaluated expression levels of the identified mitochondrial iron importers, mitoferrin 1 and 2 (42), but the transcript levels of these genes were not significantly changed (data not shown). It remains to be investigated how the patient cells overaccumulate iron in mitochondria, but many unannotated candidates for this process are contained within the array results. Mitochondrial ferritin (FTMT), which is thought to prevent reactive oxygen species and protect mitochondrial [Fe-S] enzymes (43), was observed to be increased in erythroblasts from SA patients (44) and in erythroblasts derived from the GLRX5-deficient patient (C. Camaschella et al., unpublished data). However, FTMT mRNA levels were decreased 6-fold in fibroblasts derived from this GLRX5deficient SA patient (Figure 7C), suggesting that regulation 

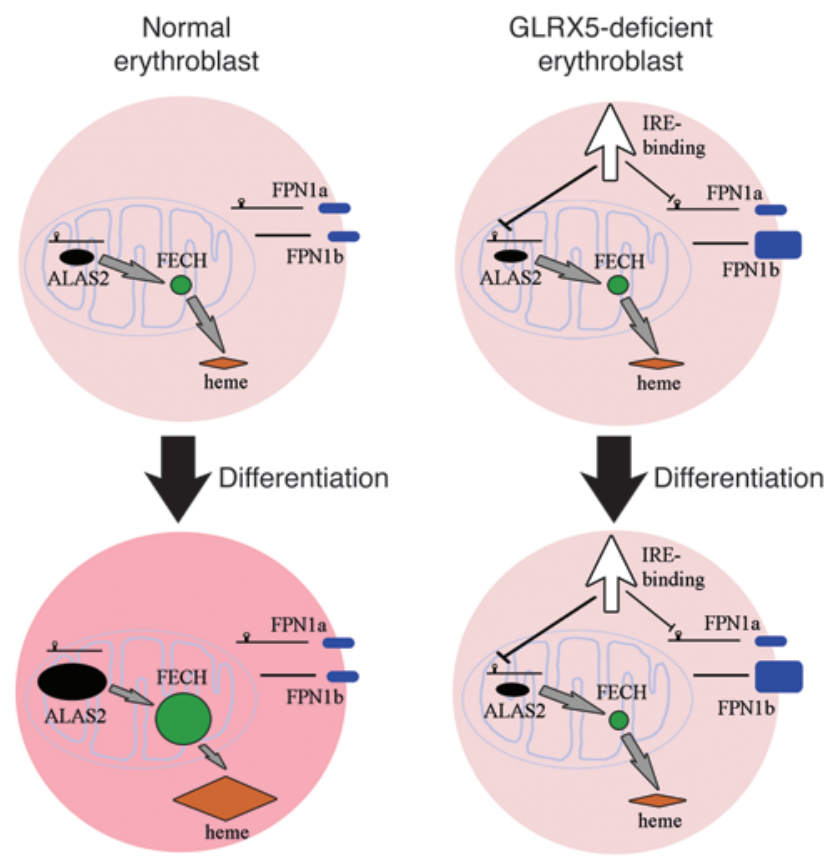

Figure 9

A working model to describe how GLRX5 deficiency in erythroblasts impairs heme biosynthesis, depletes cytosolic iron, and potentially causes SA. GLRX5 contributes to [Fe-S] cluster biogenesis and mitochondrial iron homeostasis in every tissue. Like many other [Fe-S] synthetic proteins, the deficiency of GLRX5 leads to mitochondrial iron accumulation. As reported in this study, GLRX5 has an additional role in erythropoiesis. In normal erythroblasts, ALAS2 and FECH are upregulated to generate large amounts of heme during erythropoiesis or DMSO-induced differentiation. GLRX5 is required to maintain mitochondrial iron homeostasis, which then allows normal ALAS2 protein translation and supports formation of FECH holo-protein. In GLRX5-deficient erythroblasts, GLRX5 deficiency impairs mitochondrial [Fe-S] biogenesis, induces relative cytosolic iron depletion, and activates IRE-binding activity of both IRP1 and IRP2. ALAS2 is repressed by IRPs; FECH is degraded in the absence of [Fe-S] clusters, and heme biosynthesis is accordingly impaired. In addition, the increased ferroportin (FPN1) generated from a non-IRE containing transcript (FPN1b) may deplete cytosolic iron in the GLRX5-deficient erythroblasts. GLRX5-deficient nonerythroblasts do not express ALAS2 and do not need much FECH to synthesize heme. They also express FPN1a, a transcript that contains an IRE, and is subject to translation repression from activation of IRE-binding activity. Therefore, the disturbance to heme synthesis is minimal, and nonhematopoietic tissues do not demonstrate significant phenotypes.

may vary among different tissues. Transcription of both heme oxygenase 1 (HMOX1) and ferritin can be induced in response to stresses such as oxidative stress and heavy metals (45). Our analysis revealed that HMOX1 transcripts were increased approximately 15-fold and L-ferritin transcripts were increased 2.3-fold in patient cells (Figure 7D). Together, the enhanced expression of these genes suggested that oxidative stress developed in patient cells. Interestingly, the expression of the iron exporter ferroportin (FPN1) was increased 5-fold in patient cells (Figure 7D), and inspection of the FPN1A promoter revealed that it contained several transcription factor cassette motifs in common with the promoter of HMOX1, including the Genomatix-defined transcription modules NR2F SP1F 01 (46), KLFS KLFS NFKB 01 (47), and CREB EBOX 01 (48). These transcription modules share a common feature of being involved in stress responses, and their presence in the ferroportin promoter suggests that ferroportin is among the genes activated by some classical stresses. Since the role of FPN1 is to export iron out of the cell, the increased FPN1 expression perhaps contributes to the iron deficiency in cytosol observed in GLRX5-deficient patient cells.

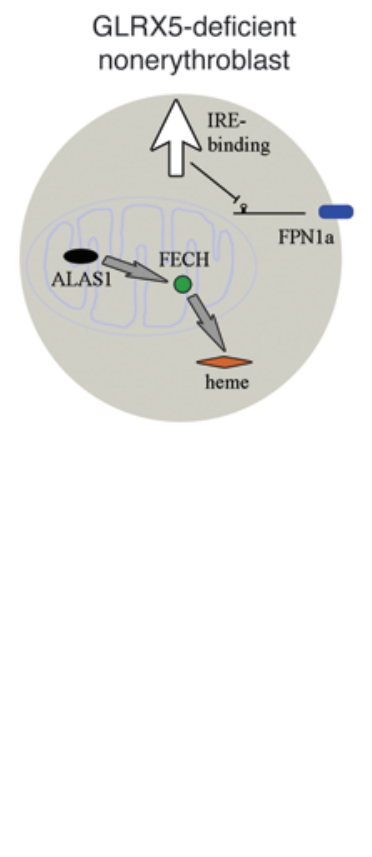

Increased FPN1 expression in part accounts for the erythroid-specific phenotype of GLRX5 deficiency. To determine whether the protein level of FPN1 was indeed increased in GLRX5-depleted cells, we performed FPN1 Western blots after GLRX5 siRNA treatment on cells from the erythroid cell line K562 and the nonerythroid cell line HeLa S3. GLRX5 protein levels were decreased to undetectable levels by siRNA treatment in both cell lines (Figure 8A). FPN1 protein levels increased about 1.5-fold in GLRX5-depleted erythroid cells from the K562 cell line (Figure 8A), whereas FPN1 protein levels were not significantly altered by GLRX5 depletion in the nonerythroid cell line HeLa S3 (Figure 8A). qRT-PCR data revealed that the total FPN1 transcripts were increased 2- to 3-fold in GLRX5-depleted K562 cells (Figure 8B), similar to the increase measured in the patient fibroblast cells (Figure 7D). Therefore, the FPN1 mRNA levels increased in multiple cell types, but FPN protein levels increased only in erythroid precursors. This discrepancy is likely due to the presence of a newly identified FPN1 mRNA variant, FPN1b, in erythroid precursors. By alternative transcription and splicing, the FPN1 gene expresses 2 mRNAs, FPN1a, which contains a $5^{\prime}$ terminal IRE and is translationally repressed by IRP proteins, and FPN1b, which does not contain the IRE and therefore evades the IRP repression (49). Thus far, the FPN1b transcript has been observed only in erythroid cells and duodenum. Our qRT-PCR results indicated that FPN1b was abundantly expressed in K562 cells and was increased 1.5- to 2-fold in GLRX5-depleted cells (Figure 8B). Since this transcript cannot be repressed by IRPs, it allows FPN1 protein levels to increase in erythroid cells even under GLRX5 depletion, which could worsen the relative iron deficiency in cytosol. Thus, the tissue-specific increase of FPN1b levels may in part account for the erythroid phenotype caused by GLRX5 deficiency by mediating a real increase in ferroportin expression.

\section{Discussion}

Here we have shown that GLRX5 is a mitochondrial protein required for iron-sulfur cluster biogenesis. GLRX5 can assemble a [2Fe-2S] cluster when GSH ligands are present in vitro, and it may function as a [2Fe-2S] donor in vivo. GLRX5 knockdown experiments in HeLa cells revealed that diminished mitochondrial aconitase activity was associated with mitochondrial iron overload and markedly increased levels of IRP2, an indication that cytosolic iron levels were decreased. In erythroid cells, GLRX5 knockdown resulted in decreased expres- 
sion of ferritin, ALAS2, and FECH. ALAS2 catalyzes the first reaction of heme biosynthesis in erythroid cells, and FECH catalyzes the last, and decreased ALAS2 and FECH expression were associated with decreased heme content. Activation of IRE-binding activity was demonstrated in fibroblasts from a patient with a splicing mutation that rendered GLRX5 protein undetectable both in lymphoblasts and fibroblasts. Increased IRE-binding activity repressed ferritin translation in several cell types and was also expected to repress ALAS2 translation in erythroid cells because ALAS2 contains an IRE in its 5' UTR. As expected, but not previously directly observed, ALAS2 protein levels were reduced in GLRX5-deficient erythroid cells. In addition, FECH levels were diminished in erythroid cells. We speculate that GLRX5 may be required to donate the [2Fe-2S] cluster to human $\mathrm{FECH}$, and recent work has demonstrated that FECH is unstable when it does not acquire its [2Fe-2S] cluster (18). FECH levels were diminished in lymphoblasts from the patient, but not in fibroblasts from the same patient, indicating some tissue specificity in FECH stability when [Fe-S] biogenesis is impaired.

In GLRX5 knockdown experiments, we showed that mitochondrial aconitase activity was diminished by GLRX5 siRNA treatment, but cytosolic aconitase activity was not significantly changed. These results implied that GLRX5 has a direct role in mitochondrial [Fe-S] biogenesis. We hypothesize that GLRX5-deficient cells fail to correctly assess mitochondrial iron status, and they therefore import more iron into mitochondria or export less, leading to mitochondrial iron overload and concomitant cytosolic iron deficiency. Under cytosolic iron deficiency, IRP2 levels are expected to increase, as was observed, and eventually cytosolic aconitase activity would decrease as a result of cytosolic iron depletion and loss of the iron-sulfur cluster required for aconitase activity. IRP1 is a bifunctional protein that converts from activity as a cytosolic aconitase when it contains an intact [4Fe-4S] cluster to an IRE-binding protein when the cluster is absent, and IRE-binding activity of IRP1 would accordingly increase in GLRX5-deficient cells. In fibroblasts from the GLRX5-deficient patient, we observed decreased cytosolic aconitase activity and increased IRE-binding activity in a gel shift assay, as expected.

Although the proteins of the glutaredoxin family are suggested to perform multiple functions, including reduction of disulfide bonds and de/glutathionylation (28), the exact function of GLRX5 in $[\mathrm{Fe}-\mathrm{S}]$ biogenesis has not yet been fully characterized. Based on prior experiments in the yeast model system, some have suggested that GLRX5 regulates activities of IscS and IscU in the [Fe-S] machinery by addition to or removal of GSH groups from active site cysteines $(30,50)$, whereas others concluded that GLRX5 is involved in an [Fe-S] cluster delivery from scaffold IscU to target proteins (51). Recently, a number of glutaredoxin proteins were found to ligate $[2 \mathrm{Fe}-2 \mathrm{~S}]$ clusters in vitro. The discovery that plant monothiol GRX bind a [2Fe-2S] cluster has led to the proposal that these monothiol GRX function as scaffold proteins that deliver [2Fe-2S] clusters to specific recipient target proteins (34). Our observation that a $[2 \mathrm{Fe}-2 \mathrm{~S}]$ bridging cluster can be reconstituted on human GLRX5 is consistent with this hypothesis. In the reconstituted holo-GLRX5, the catalytic residue C67 and external GSH are required to ligate the [2Fe-2S] cluster between 2 monomers. Point mutagenesis revealed that K59, T108, and D123 residues were likely involved in the docking of the GSH ligand to GLRX5 protein. Thus, as suggested for its plant counterparts (AtGrxS14 and AtGrxS16) (34), GLRX5 could function as a potential scaffold, delivering the preassembled [2Fe-2S] cluster to target proteins.
A possible cause for the observation that relative cytosolic iron deficiency occurs in conjunction with compromise of [Fe-S] biogenesis (52) was revealed by gene profiling, in which we observed that HMOX1 and ferroportin transcript levels increased markedly in GLXR-deficient cells, likely in response to the stress associated with functional failure of [Fe-S] proteins. With the disruption in mitochondrial iron homeostasis caused by defective [Fe-S] assembly in yeast, reactive iron can compete with manganese for binding to SOD2, and iron binding to eukaryotic SOD2 irreversibly inactivates the SOD enzyme, thus leading to oxidative stress (53, 54). Diminished expression of FTMT mRNA in GLRX5-deficient fibroblasts could also contribute further to the damage from mitochondrial iron overload by failing to provide a protein that can sequester mitochondrial iron. Cytosolic iron deficiency may contribute to the slow-growth phenotype observed in Caenorbabditis elegans that lack GLRX5 and in fibroblasts and lymphoblasts from the GLRX5-deficient patients. Notably, we demonstrated here that the slow growth of the GLRX5-deficient patient fibroblasts was reversed by GLRX5 expression.

Hereditary mutations of several different [Fe-S] synthetic genes are known to cause human disorders (19), including mutations in the gene of the proposed iron donor, frataxin in FRDA, in the iron-sulfur cluster scaffold protein ISCU in patients with hereditary myopathy, and in the SA patient discussed in this paper. Although these mutations affect the same basic process, the manifestations of disease differ greatly; heart and brain are the primary affected tissues in FRDA, whereas skeletal muscle is affected in ISCU myopathy, and erythroid cells are adversely affected in the GLRX5-deficient patient, raising the question of why symptoms and affected tissues are so variable. The mutations of ISCU myopathy and GLRX5-related SA are caused by splicing defects, whereas mutations of ABCB7 that cause inherited SA/ataxia syndrome are missense mutations. Thus, one possible reason for tissue specificity may be that splicing defects are tissue specific.

However, a tissue-specific splicing defect cannot explain the GLRX5-caused SA, since we have shown that GLRX5 is absent in both hematopoietic (lymphoblast) and nonhematopoietic (fibroblast) cells. We suggest that SA occurs with GLRX5 deficiency mainly because ALAS2, which is expressed almost exclusively in erythroid cells, contains an IRE in its 5' UTR that renders it susceptible to translational repression by IRPs. The defect in regulation of mitochondrial iron homeostasis caused by loss of GLRX5 also affects nonerythroid cells, as we demonstrated with GLRX5 knockdown experiments and in patient fibroblasts, but many effects may remain below the threshold for clinical detection, whereas repression of ALAS2 in erythroid cells causes profound symptoms (Figure 9).

We propose that the tissue-specific phenotype of human GLRX5 deficiency depends not only on the erythroid-specific expression of ALAS2, which contains an IRE and is translationally repressed by IRPs, but also on the expression of ferroportin $1 \mathrm{~b}$, a ferroportin transcript that lacks an IRE and cannot be repressed by IRPs. Increased expression of both FPN transcripts likely occurs in response to stress caused by mitochondrial failure to synthesize iron-sulfur clusters and to correctly regulate iron homeostasis, but the expression of ferroportin $1 \mathrm{~b}$ then exacerbates the cytosolic iron depletion and further misregulates iron homeostasis in erythroid precursors. Thus, GLRX5 deficiency would be expected to impair [Fe-S] biogenesis in many tissues, but only in erythroid precursors would the unique profile of IRP targets cause a clinically significant syndrome. We propose that as more [Fe-S] syn- 
thetic components are identified and characterized, more insights into possible causes of tissue specificity for diseases of Fe-S biogenesis may be discovered.

\section{Methods}

Cell culture. Human epithelial HeLa S3, HeLa cells, skin fibroblast cells (ATCC), and fibroblast cells derived from the GLRX5 mutation patient (24) were maintained in DMEM containing 10\% FBS, 2 mM L-glutamine, and $100 \mathrm{U} / \mathrm{ml}$ PenStrep. The patient gave informed consent, and studies were approved by the institutional review board of the Università VitaSalute. Human erythroid K562 cells (ATCC) and lymphoblast cells were maintained in RPMI 1640 medium containing 10\% FBS, 2 mM L-glutamine, and $100 \mathrm{U} / \mathrm{ml}$ PenStrep. Lymphoblast cells derived from the GLRX5 mutation patient (24) were maintained in RPMI 1640 medium (Lonza) containing 20\% FBS, 2 mM L-glutamine, and 100 U/ml PenStrep.

$R N A i$. Four siRNA oligonucleotides were synthesized by QIAGEN and used to transfect cells. The target sequences in GLRX5 genes were as follows: oligo 1: AAGAAGGACAAGGTGGTGGTC; oligo 2: AAGTGTACCTCAATGGCGAGT; oligo 3: CTGGTGTTCGGGCTAAGAATA; and oligo 4: CTGTATTATGATATTGCTGTA. A nonspecific oligo (QIAGEN) that is not complementary to any human genes was used as a negative control. HeLa S3 cells were transfected with lipofectamine 2000 (Invitrogen) following the manufacturer's protocol. To transfect K562 cells, HiPerFect reagent (QIAGEN) was used following the manufacturer's protocol.

Immunofluorescence staining and enhanced Perls DAB iron staining. HeLa cells were grown on the coverslip in a 6-well plate to $40 \%-60 \%$ confluency. Rinsed in PBS buffer, the cells were fixed with $4 \%$ paraformaldehyde and $0.1 \%$ Triton X-100, and blocked with $10 \%$ FBS. Cells were subsequently incubated with primary antibodies (GLRX5, 1:1500 dilution; and Tom20, 1:500 dilution) and secondary antibodies (Alexa Fluor 488-conjugated goat anti-rabbit antibody and Alexa Fluor 546-conjugated goat antimouse antibody) separated by thorough PBS rinses. Mounted slides were examined under confocal microscope LSM 510 (Zeiss). Enhanced Perls DAB cellular iron staining was performed as described (9).

Enzyme assay, transcript, and iron quantitation. Aconitase activity in-gel assay and band-shift assay were performed as described (9). XO activity was assayed using Amplex Red XO Assay Kit (Molecular Probes). Complex I activity and quantity were assayed using Human Complex I Activity Assay Kit and Quantity Assay Kit (MitoSciences). Gene transcripts were quantified by real-time RT-PCR. Total RNA was isolated using TRIzol reagent (Invitrogen) and reverse transcribed to cDNA using the High Capacity cDNA Reverse Transcription Kit (Applied Biosystems). Real-time PCR was performed using CYBR green PCR Master Mix (Applied Biosystems) on ABI Prism 7000 Sequence Detection System (Applied Biosystems). Primers used are shown in Supplemental Table 1. Cellular heme was quantified using QuantiChrom Heme Assay Kit (BioAssay Systems). Cellular nonheme iron was quantified using QuantiChrom Iron Assay Kit (BioAssay Systems). Subcellular fractions of mitochondria and cytosol were prepared using Mitochondria Isolation Kit For Mammalian Cells (Pierce).

GLRX5 protein purification and site-directed mutagenesis. The mature sequence of human GLRX5 was cloned into pET28a vector (Novagen) with an N-terminal His6 tag. The resulting plasmid was transformed into BL21 cells for the expression of recombinant protein. His-tagged GLRX5 was purified as described (55). The His6 tag was cleaved by Thrombin Cleavage Capture Kit (Novagen). The purified recombinant GLRX5 was used in raising polyclonal antibody and in vitro $[\mathrm{Fe}-\mathrm{S}]$ reconstitution. The amino acid substitution was performed using QuikChange Site-Directed Mutagenesis Kit (Stratagene). Mutant GLRX5 proteins were expressed and purified as described above. Primers used are shown in Supplemental Table 2.
Anaerobic [Fe-S] reconstitution. [Fe-S] clusters were reconstituted into purified GLRX5 proteins in an anaerobic chamber. The reconstitution mixture contained $200 \mu \mathrm{M}$ GLRX5 protein, $5 \mathrm{mM}$ DTT, $2 \mathrm{mM} \mathrm{GSH}, 2 \mathrm{mM}$ lithium sulfide (Sigma-Aldrich), and $2 \mathrm{mM}$ ferrous chloride. After 2 hours incubation at room temperature, protein was purified by a PD-10 desalting column (GE Healthcare) and subsequently by a HiTrap Q HP column (GE Healthcare). All buffers for purification contained $1 \mathrm{mM} \mathrm{GSH}$. The finally recovered holo-GLRX5 fraction was concentrated by Amicon Ultra Centrifugal Filter Device (Millipore). UV-vis spectrophotometry of holoGLRX5 was performed using NanoDrop ND-1000 (Thermal Scientific). Mossbauer analysis of holo-GLRX5 was performed as described (11).

Transfection and transduction. To rescue patient fibroblast cells, a WT GLRX5 sequence was cloned into pCMV-3tag plasmid (Stratagene). The resulting plasmid pCMV-GLRX5 was introduced into patient cells grown on a 24-well plate by Neon Transfection System (Invitrogen). A WT GLRX5 sequence was also cloned to an HIV1 viral vector. The resulting virus HIV1GLRX5 expresses both GLRX5 and GFP in a single mRNA with an IRES sequence between them, allowing separate translation of the 2 proteins. The viral vector was used to transduce patient cells as described.

Antibodies. GLRX5 antibody was raised against purified recombinant GLRX5 protein in rabbit (Covance) and affinity purified using pure proteins coupled on the medium of CNBr-activated sepharose 4B (Amersham Pharmacia). Antibodies for $\mathrm{m}$-aconitase, FECH, IRP1, IRP2, and H-ferritin were as described $(9,56)$. ALAS2 antibody was raised as described (57) and affinity purified using recombinant ALAS2 protein. Other antibodies were $\alpha$-tubulin (Sigma-Aldrich), SOD2 (Abcam), ABCB7 (Santa Cruz Biotechnology Inc.), Tom 20 (Santa Cruz Biotechnology Inc.), Alexa Fluor 488conjugated goat anti-rabbit antibody, and Alexa Fluor 546-conjugated goat anti-mouse antibody (Molecular Probes).

In situ bybridization of mouse tissues. Mouse full-length GLRX5 sequence in PCMV-SPORT6.1 (Invitrogen) was linearized at either end and used to prepare sense and anti-sense probes. In situ hybridization was performed on mouse tissues as described (56).

Bioinformatics. Transcription module analysis of gene promoters was performed using Genomatix tools (www.genomatix.de). Tissue-specific gene expression analysis was performed using an online tool (BioGPS; http://biogps.gnf.org/\#goto=welcome). The gene knockdown phenotype was analyzed using a worm database (www.wormbase.org).

Statistics. Unless otherwise indicated, results are expressed as mean $\pm \mathrm{SD}$ of 3 independent experiments. $P$ values were determined by 2 -tailed Student's $t$ test. A $P$ value less than 0.05 was considered significant.

\section{Acknowledgments}

We thank W.H. Tong, D. Crooks, H. Uhrigshardt, D. Zhang, and S. Cooperman for providing antibodies and cell lines. We also thank P. Kim and G. Patterson for help in confocal microscopy and W. Chen of genomics core laboratory at the National Institute of Diabetes and Digestive and Kidney Diseases (NIDDK) for microarray analysis. This work was supported by the intramural program of the National Institute of Child Health and Human Development (to T. Rouault) and by a grant from Roche Foundation for Anemia Research (ROFAR) (to C. Camaschella).

Received for publication July 1, 2009, and accepted in revised form January 20, 2010.

Address correspondence to: Tracey A. Rouault, Molecular Medicine Program, National Institute of Child Health and Human Development, NIH, Bethesda, Maryland 20892, USA. Phone: 301.496.7060; Fax: 301.402.0078; E-mail: trou@helix.nih.gov. 
1. Beinert H, Holm RH, Munck E. Iron-sulfur clusters: nature's modular, multipurpose structures. Science. 1997;277(5326):653-659.

2. Beinert H. Iron-sulfur proteins: ancient structures, still full of surprises. J Biol Inorg Chem. 2000 $5(1): 2-15$.

3. Johnson DC, Dean DR, Smith AD, Johnson MK. Structure, function, and formation of biological iron-sulfur clusters. Annu Rev Biochem. 2005;74:247-281.

4. Lill R, Muhlenhoff U. Maturation of iron-sulfur proteins in eukaryotes: mechanisms, connected processes, and diseases. Annu Rev Biochem. 2008;77:669-700.

5. Ye H, Pilon M, Pilon-Smits EA. CpNifS-dependent iron-sulfur cluster biogenesis in chloroplasts. New Pbytol. 2006;171(2):285-292.

6. Rouault TA, Tong WH. Iron-sulphur cluster biogenesis and mitochondrial iron homeostasis. Nat Rev Mol Cell Biol. 2005;6(4):345-351.

7. Lill R, Muhlenhoff U. Iron-sulfur-protein biogenesis in eukaryotes. Trends Biochem Sci. 2005;30(3):133-141.

8. Tong WH, Rouault T. Distinct iron-sulfur cluster assembly complexes exist in the cytosol and mitochondria of human cells. EMBO J. 2000;19(21):5692-5700.

9. Tong WH, Rouault TA. Functions of mitochondrial ISCU and cytosolic ISCU in mammalian ironsulfur cluster biogenesis and iron homeostasis. Cell Metab. 2006;3(3):199-210.

10. Land T, Rouault TA. Targeting of a human iron-sulfur cluster assembly enzyme, nifs, to different subcellular compartments is regulated through alternative AUG utilization. Mol Cell. 1998;2(6):807-815.

11. Tong WH, Jameson GN, Huynh BH, Rouault TA Subcellular compartmentalization of human Nfu, an iron-sulfur cluster scaffold protein, and its ability to assemble a [4Fe-4S] cluster. Proc Natl Acad Sci US A. 2003;100(17):9762-9767.

12. Rouault TA. The role of iron regulatory proteins in mammalian iron homeostasis and disease. Nat Chem Biol. 2006;2(8):406-414.

13. Volz K. The functional duality of iron regulatory protein 1. Curr Opin Struct Biol. 2008;18(1):106-111.

14. Eisenstein RS. Iron regulatory proteins and the molecular control of mammalian iron metabolism. Annu Rev Nutr. 2000;20:627-662.

15. Pantopoulos K. Iron metabolism and the IRE/IRP regulatory system: an update. Ann N Y Acad Sci. 2004;1012:1-13.

16. Wallander ML, Leibold EA, Eisenstein RS. Molecular control of vertebrate iron homeostasis by iron regulatory proteins. Biochim Biophys Acta. 2006;1763(7):668-689.

17. Ajioka RS, Phillips JD, Kushner JP. Biosynthesis of heme in mammals. Biochim Biopbys Acta. 2006;1763(7):723-736.

18. Crooks DR, Ghosh MC, Haller RG, Tong WH, Rouault TA. Post-translational stability of the heme biosynthetic enzyme ferrochelatase is dependent on iron availability and intact iron-sulfur cluster assembly machinery. Blood. 2009;115(4):860-869.

19. Rouault TA, Tong WH. Iron-sulfur cluster biogenesis and human disease. Trends Genet. 2008;24(8):398-407.

20. Puccio H, Koenig M. Friedreich ataxia: a paradigm for mitochondrial diseases. Curr Opin Genet Dev. 2002;12(3):272-277.

21. Puccio $\mathrm{H}$, et al. Mouse models for Friedreich ataxia exhibit cardiomyopathy, sensory nerve defect and Fe-S enzyme deficiency followed by intramitochon- drial iron deposits. Nat Genet. 2001;27(2):181-186.

22. Mochel F, et al. Splice mutation in the iron-sulfur cluster scaffold protein ISCU causes myopathy with exercise intolerance. Am J Hum Genet. 2008;82(3):652-660.

23. Olsson A, Lind L, Thornell LE, Holmberg M. Myopathy with lactic acidosis is linked to chromosome 12q23.3-24.11 and caused by an intron mutation in the ISCU gene resulting in a splicing defect. Hum Mol Genet. 2008;17(11):1666-1672

24. Camaschella $C$, et al. The human counterpart of zebrafish shiraz shows sideroblastic-like microcytic anemia and iron overload. Blood. 2007; 110(4):1353-1358.

25. Camaschella C. Recent advances in the understanding of inherited sideroblastic anaemia. BrJ Haematol. 2008;143(1):27-38.

26. Cavadini $P$, et al. RNA silencing of the mitochondrial ABCB7 transporter in HeLa cells causes an iron-deficient phenotype with mitochondrial iron overload. Blood. 2007;109(8):3552-3559.

27. Guernsey DL, et al. Mutations in mitochondrial carrier family gene SLC25A38 cause nonsyndromic autosomal recessive congenital sideroblastic anemia. Nat Genet. 2009;41(6)(6):651-653.

28. Herrero E, de la Torre-Ruiz MA. Monothiol glutaredoxins: a common domain for multiple functions. Cell Mol Life Sci. 2007;64(12):1518-1530.

29. Lillig CH, Berndt C, Holmgren A. Glutaredoxin systems. Biochim Biophys Acta. 2008;1780(11):1304-1317.

30. Rodriguez-Manzaneque MT, Tamarit J, Belli G, Ros J, Herrero E. Grx5 is a mitochondrial glutaredoxin required for the activity of iron/sulfur enzymes. Mol Biol Cell. 2002;13(4):1109-1121.

31. Witte S, Villalba M, Bi K, Liu Y, Isakov N, Altman A. Inhibition of the c-Jun $\mathrm{N}$-terminal kinase/ AP-1 and NF-kappaB pathways by PICOT, a novel protein kinase $\mathrm{C}$-interacting protein with a thioredoxin homology domain. J Biol Chem. 2000;275(3):1902-1909.

32. Wingert RA, et al. Deficiency of glutaredoxin 5 reveals Fe-S clusters are required for vertebrate haem synthesis. Nature. 2005;436(7053):1035-1039.

33. Tong WH, Rouault TA. Metabolic regulation of citrate and iron by aconitases: role of iron-sulfur cluster biogenesis. Biometals. 2007;20(3-4):549-564.

34. Bandyopadhyay S, et al. Chloroplast monothiol glutaredoxins as scaffold proteins for the assembly and delivery of $[2 \mathrm{Fe}-2 \mathrm{~S}]$ clusters. $E M B O \mathrm{~J}$. 2008;27(7):1122-1133.

35. Picciocchi A, Saguez C, Boussac A, Cassier-Chauvat C, Chauvat F. CGFS-type monothiol glutaredoxins from the cyanobacterium Synechocystis PCC6803 and other evolutionary distant model organisms possess a glutathione-ligated $[2 \mathrm{Fe}-2 \mathrm{~S}]$ cluster. Biochemistry. 2007;46(51):15018-15026.

36. Rouhier N, et al. Functional, structural, and spectroscopic characterization of a glutathione-ligated [He-2S] cluster in poplar glutaredoxin C1. Proc Natl Acad Sci U S A. 2007;104(18):7379-7384.

37. Lillig $\mathrm{CH}$, et al. Characterization of human glutaredoxin 2 as iron-sulfur protein: a possible role as redox sensor. Proc Natl Acad Sci US A. 2005;102(23):8168-8173.

38. Hinchliffe P, Sazanov LA. Organization of ironsulfur clusters in respiratory complex I. Science. 2005;309(5735):771-774.

39. Dailey HA, Finnegan MG, Johnson MK. Human ferrochelatase is an iron-sulfur protein. Biochemistry. 1994;33(2):403-407.

40. Rouault TA. Linking physiological functions of iron. Nat Chem Biol. 2005;1(4):193-194.
41. Uchida N, et al. Development of a human immunodeficiency virus type 1-based lentiviral vector that allows efficient transduction of both human and rhesus blood cells. J Virol. 2009;83(19):9854-9862.

42. Paradkar PN, Zumbrennen KB, Paw BH, Ward $\mathrm{DM}$, Kaplan J. Regulation of mitochondrial iron import through differential turnover of mitoferrin 1 and mitoferrin 2. Mol Cell Biol. 2009; 29(4):1007-1016.

43. Campanella A, Rovelli E, Santambrogio P, Cozzi A, Taroni F, Levi S. Mitochondrial ferritin limits oxidative damage regulating mitochondrial iron availability: hypothesis for a protective role in Friedreich ataxia. Hum Mol Genet. 2009;18(1):1-11.

44. Cazzola M, et al. Mitochondrial ferritin expression in erythroid cells from patients with sideroblastic anemia. Blood. 2003;101(5):1996-2000.

45. Vile GF, Basu-Modak S, Waltner C, Tyrrell RM. Heme oxygenase 1 mediates an adaptive response to oxidative stress in human skin fibroblasts. Proc Natl Acad Sci U S A. 1994;91(7):2607-2610.

46. Ghadially H, Ross XL, Kerst C, Dong J, Reske-Kunz $\mathrm{AB}$, Ross R. Differential regulation of CCL22 gene expression in murine dendritic cells and B cells. J Immunol. 2005;174(9):5620-5629.

47. Clerk A, Kemp TJ, Zoumpoulidou G, Sugden PH. Cardiac myocyte gene expression profiling during $\mathrm{H} 2 \mathrm{O} 2$-induced apoptosis. Physiol Genomics. 2007;29(2):118-127.

48. Pan L, et al. Critical roles of a cyclic AMP responsive element and an E-box in regulation of mouse renin gene expression. J Biol Chem. 2001; 276(49):45530-45538.

49. Zhang DL, Hughes RM, Ollivierre-Wilson $\mathrm{H}$, Ghosh MC, Rouault TA. A ferroportin transcript that lacks an iron-responsive element enables duodenal and erythroid precursor cells to evade translational repression. Cell Metab. 2009;9(5):461-473.

50. Rodriguez-Manzaneque MT, Ros J, Cabiscol E, Sorribas A, Herrero E. Grx5 glutaredoxin plays a central role in protection against protein oxidative damage in Saccharomyces cerevisiae. Mol Cell Biol. 1999;19(12):8180-8190.

51. Muhlenhoff U, Gerber J, Richhardt N, Lill R. Components involved in assembly and dislocation of iron-sulfur clusters on the scaffold protein Isu1p. EMBO J. 2003;22(18):4815-4825.

52. Li K, Besse EK, Ha D, Kovtunovych G, Rouault TA. Iron-dependent regulation of frataxin expression: implications for treatment of Friedreich ataxia. Hum Mol Genet. 2008;17(15):2265-2273.

53. Culotta VC, Yang M, O'Halloran TV. Activation of superoxide dismutases: putting the metal to the pedal. Biochim Biophys Acta. 2006;1763(7):747-758.

54. Luk E, Yang M, Jensen LT, Bourbonnais Y, Culotta VC. Manganese activation of superoxide dismutase 2 in the mitochondria of Saccharomyces cerevisiae. J Biol Chem. 2005;280(24):22715-22720.

55. Ye H, Abdel-Ghany SE, Anderson TD, Pilon-Smits EA, Pilon M. CpSufE activates the cysteine desulfurase $\mathrm{CpNifS}$ for chloroplastic Fe-S cluster formation. J Biol Chem. 2006;281(13):8958-8969.

56. Meyron-Holtz EG, et al. Genetic ablations of iron regulatory proteins 1 and 2 reveal why iron regulatory protein 2 dominates iron homeostasis. EMBOJ. 2004;23(2):386-395.

57. Cooperman SS, Meyron-Holtz EG, OlivierreWilson H, Ghosh MC, McConnell JP, Rouault TA. Microcytic anemia, erythropoietic protoporphyria, and neurodegeneration in mice with targeted deletion of iron-regulatory protein 2. Blood. 2005;106(3):1084-1091. 